\title{
HUMAN-INSPIRED MOTION PRIMITIVES AND TRANSITIONS FOR BIPEDAL ROBOTIC LOCOMOTION IN DIVERSE TERRAIN
}

\author{
A Thesis \\ by \\ HUIHUA ZHAO
}

\begin{abstract}
Submitted to the Office of Graduate and Professional Studies of Texas A\&M University in partial fulfillment of the requirements for the degree of

MASTER OF SCIENCE
\end{abstract}

\author{
Chair of Committee, Aaron D. Ames \\ Committee Members, Reza Langari \\ Shankar P. Bhattacharyya \\ Sivakumar Rathinam \\ Head of Department, Andreas Polycarpou
}

May 2015

Major Subject: Mechanical Engineering

Copyright 2015 Huihua Zhao 


\begin{abstract}
This thesis presents a control design approach, which uses human data in the development of bipedal robotic control techniques for multiple locomotion behaviors. Insight into the fundamental behaviors of human locomotion is obtained through the examination of experimental human data for level walking, stair ascending, stair descending and running. Specifically, it is shown that certain outputs of the human, independent of locomotion terrain, can be characterized by a single function, termed the extended canonical human function. Through feedback linearization, humaninspired locomotion controllers are leveraged to drive the outputs of the simulated robot, via the extended canonical human function, to the outputs from human locomotion. An optimization problem, subject to the constraints of partial hybrid zero dynamics, is presented which yields parameters of these controllers that provide the best fit to human data while simultaneously ensuring stability of the controlled bipedal robot. The resulting behaviors are stable locomotion on flat ground, upstairs, downstairs and running — these four locomotion modes are termed "motion primitives". A second optimization is presented, which yields controllers that evolve the robot from one motion primitive to another - these modes of locomotion are termed "motion transitions". A directed graph consisting these motion primitives and motion transitions has been constructed for the stable motion planning of bipedal locomotion. A final simulation is given, which shows the controlled evolution of a robotic biped as it transitions through each mode of locomotion over a pyramidal staircase.
\end{abstract}




\section{DEDICATION}

To my mom and my girlfriend, for never ceasing in their love, encouragement, and support as I have grown through my life. 


\section{ACKNOWLEDGEMENTS}

I would first like to thank my mom for her endless and unconditional support during my entire education, for inspiring me always chasing the best. I would like to thank my girlfriend, Huanlin for her continuously encouraging and supporting, for her accompanying through all these tough but fruitful days. I would not make this far without their support.

I would put my great attitude to my advisor, Dr. Aaron Ames for his guidance and support throughout my research work, and for his enthusiasm for theoretically succinct that points me the right way to pursue. I would also have my thanks to my friends and teammates, Shishir Yadukumar and Matthew Powell for sharing valuable ideas and insights with me that finally leads me to accomplish this much. I would also extend my sincere gratitude to my advisory committee Dr. Langari, Dr. Bhattacharyya and Dr. Rathinam for their keen supports in making this work a valuable literature. It's their courses that I have taken inspire my research and lead me through the puzzles. 


\section{TABLE OF CONTENTS}

Page

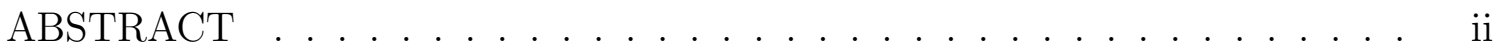

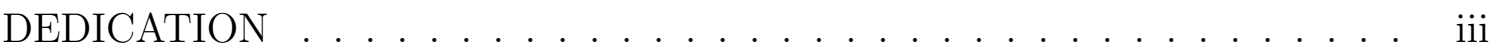

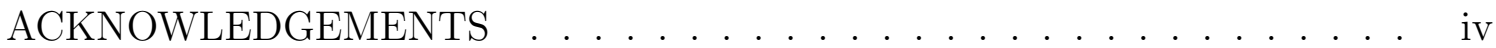

TABLE OF CONTENTS . . . . . . . . . . . . . . . . v v

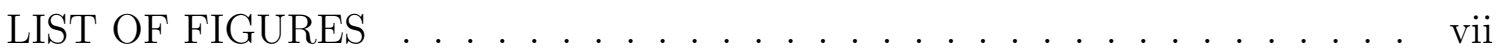

LIST OF TABLES ....................... . . . ix

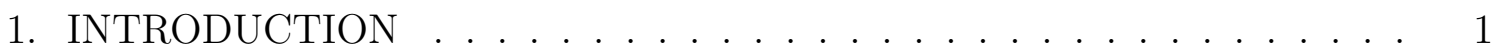

2. HUMAN LOCOMOTION DATA ................... 5

2.1 Human Locomotion Data . . . . . . . . . . . . . . . . . . . . . 5

2.2 Automated Domain Breakdown . . . . . . . . . . . . . . . . 6

2.3 Extended Canonical Human Function . . . . . . . . . . . . . . . 6

2.4 Human Outputs . . . . . . . . . . . . . . . . . . . . 10

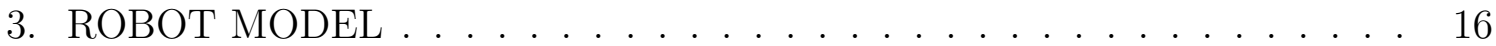

3.1 Continuous Dynamics . . . . . . . . . . . . . . . . . . . 16

3.2 Discrete Dynamics . . . . . . . . . . . . . . . . . . . . . . . 19

4. CONTROLlER DESIGN . . . . . . . . . . . . . . . . . 20

4.1 Parameterization of Time . . . . . . . . . . . . . . . 20

4.2 Controller Specification . . . . . . . . . . . . . . . 20

5. HYBRID AND META-HYBRID SYSTEMS . . . . . . . . . . . . 23

5.1 Hybrid System for the Biped . . . . . . . . . . . . . . . . 23

5.2 Meta-Hybrid Systems . . . . . . . . . . . . . . . . . . 25

6. MOTION PRIMITIVES \& TRANSITIONS . . . . . . . . . . . . . . . 28

6.1 Motion Primitives . . . . . . . . . . . . . . . . . . . . . . . . . . . . 29 
6.1.1 Motion Primitive Collection . . . . . . . . . . . . . . . . 29

6.1.2 Controller Development . . . . . . . . . . . . . . . 30

6.1.3 Partial Hybrid Zero Dynamics . . . . . . . . . . . . . . . . . 31

6.1.4 Human-Inspired Optimization . . . . . . . . . . . . . . . 32

6.1.5 Simulation Results . . . . . . . . . . . . . . . 37

6.2 Motion Transitions . . . . . . . . . . . . . . . . . . 39

6.2.1 Motion Transition Collection . . . . . . . . . . . . . . . 39

6.2.2 Controller Development . . . . . . . . . . . . . . . . . 40

6.2.3 Simulations Results . . . . . . . . . . . . . . . 45

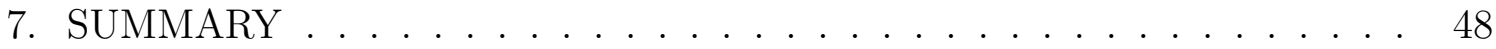

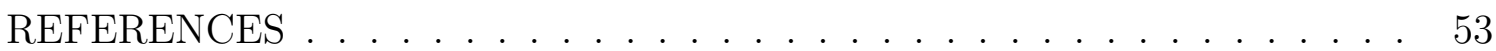




\section{LIST OF FIGURES}

2.1 Domain breakdown for walking up stairs [38]. $\ldots \ldots \ldots \ldots \ldots$

2.2 Stance knee angle data fit comparison between the ECHF and the 4th order polynomial function at the outside of the nominal operation region, which is obtained by extending $20 \%$ of the nominal operation region. The superscripts $H, E, P$ denote the human data, ECHF fitting and polynomial fitting, respectively $[38] . \ldots \ldots \ldots \ldots$

2.3 Fitted ECHF and corresponding mean human data of motion primitives $[38]$

2.4 Fitted ECHF and corresponding mean human data of motion transitions $[38] . \ldots \ldots \ldots \ldots \ldots \ldots$

2.5 Fitted CHF and corresponding mean human data of running [39]. . . 15

3.1 The modeled robot's configuration, mass and length distribution, and virtual constraints [38] . . . . . . . . . . . . . . . . 17

6.1 Graph of a meta-system representation for the five motion primitives in consideration. . . . . . . . . . . . . . . . . . . .

6.2 Snapshots from robotic locomotion simulations exhibiting the four motion primitives $[38] . \ldots \ldots \ldots \ldots \ldots \ldots$

6.3 Phase portraits for the four motion primitives. . . . . . . . . . 36

6.4 Optimized extended canonical human functions with parameters obtained by solving the optimization problem (2.6) and the corresponding mean human data of three one-phase motion primitives [38] . . .

6.5 Optimized canonical human functions with parameters obtained by solving the optimization problem (2.6) and the corresponding mean human data of the two-phase running motion primitive [39]. . . . . . 38

6.6 Optimized extended canonical human functions with parameters obtained by solving the optimization problem (2.6) and the corresponding mean human data of four motion transitions [38]. . . . . . . . . . 
6.7 Phase portraits for the combination of motion primitives and motion

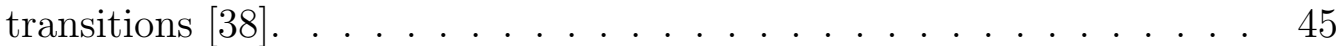

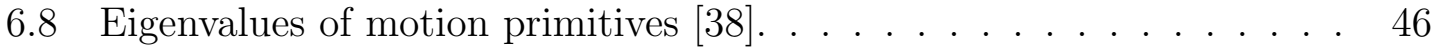

6.9 Snapshots from the simulated composition of multiple locomotion

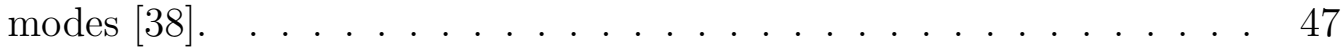

6.10 Snapshots from the simulated transitions between running and level walking. . . . . . . . . . . . . . . . . . . . . 4 47 


\section{LIST OF TABLES}

TABLE

Page

7.1 Fitted parameter values for human functions. . . . . . . . . . . . . . 49

7.2 Fitted parameter values for human functions of running. . . . . . . . 50

7.3 Optimized parameter values and cost for human functions. . . . . . . 51

7.4 Optimized parameter values and cost for human functions of running. 52 


\section{INTRODUCTION *}

Obtaining truly human-like bipedal robotic walking has been one of the most prominent goals of the humanoid robotics community. The need for functional robots, and the understanding thereof, is apparent in many fields today. In light of recent events, such as the tragic crisis at the Fukushima nuclear plant in Japan, robots are needed which can traverse dangerous terrain and work in virulent environments [1, 24]. Robots capable of human-like walking, climbing, running and all the basic modes of human locomotion, are necessary for truly successful cooperation with mankind. Moreover, achieving human-like bipedal robotic locomotion can inversely help humans understand the human body in more depth. This understanding can be leveraged to build prosthetic devices $[5,22,27]$ for lower extremity amputees, which will help them walk with a gait as natural as a healthy human. Therefore, the important and far-reaching ramifications of obtaining human-like bipedal walking have attracted many researchers from a variety of fields of study.

During three decades of study, researchers have accomplished a remarkable number of achievements on bipedal walking and climbing. Honda's ASIMO [15] has been the most popular humanoid robot around the world, yet its fundamental control scheme is to keep the center of pressure within the support polygon all the time (which is known as ZMP method). Attracted by the energy efficiency and the human-like aesthetics of passive walking [25], which was first realized by Tad McGeer, a number of robots, such as [9], [10], Flame of TU Delft, have been constructed using this basic principle. Jessy Grizzle's planar robot MABEL [33] holds the title of

\footnotetext{
*Portions of this thesis have been reprinted with permission from "Human-inspired motion primitives and transitions for bipedal robotic locomotion in diverse terrains" by H. Zhao, M. J. Powell and A. D. Ames, 2012. Optimal Control Applications and Methods.
} 
quickest bipedal robot in the world - and can also walk on uneven terrains. Most of the current research in bipedal robotics is focused on 2D locomotion, i.e., planar robots.

Taking a different approach, we leverage the knowledge of human body to develop locomotion control schemes. The quintessential model of bipedal locomotion - the human body — is the most prevalent source of information on bipedal walking. The physical human system, which utilizes 57 muscles in locomotion [28], is far too complex to replicate with current hardware and computational capabilities. However, one can construct a low-level representation of the human locomotion system. That is, from a control theorist perspective, one can view the human body as a "black box", while certain outputs of which can be represented by simple, time-based functions. Utilizing these outputs functions via the feedback linearization control method, the robot will display the same qualitative behavior as the human, despite the physical differences between the two systems. This approach is similar to the work of others in the control and biomechanical fields. By merging human data and hybrid zero dynamics, Westervelt [34] has achieved stable walking for a 5-link robot model in simulation with Bezier series fit to the human data. Work based on the same fitting method can also be found in [32]. Predictive dynamics with B-spline fit to human data has been applied to simulate normal human walking and human walking with external load [37]. Polynomial function is also one of the popular choices used to characterize outputs of human $[7,8]$. However, unlike all these functions, the method proposed in this work offers a simpler formula which can represent all the motion primitives and motion transitions with high correlations while simultaneously render more robustness outside the operation region.

In this thesis, a human-inspired controller is considered which utilizes the simple low dimensional function — extended canonical human function (ECHF) - to 
represent human locomotion. The study begins with the examination of human locomotion data that are made public by the motion capture lab in UC Berkeley. The human experimental data contains the xyz-position for flat ground walking, walking upstairs, walking downstairs and running; these data form the foundation of our control approach. Specifically, the ECHF is shown to represent the sets of data, from four modes of locomotion (walking on flat ground, going upstairs, going downstairs and running) of interest and four transition modes, with high correlation in each case. More importantly, it is found that the ECHF tends to have a more stable behavior even outside the operation region while other methods are easy to blow up if considered outside the operation window. This is very important especially for a physical robot that is vulnerable to the various environment uncertainty and disturbance. The locomotion of flat-ground, up-slope and rough terrain have been successfully achieved on a physical robot AMBER with the proposed ECHF [19]. We argue that it's the special form of the function on which the controller is based that plays a key role to the easy implementation (corresponding to the fewer parameters) and the robustness of the walking (corresponding to the better behavior outside the region of definition).

A formal construction scheme for hybrid systems - the meta-hybrid system is presented, in which a distinction is made between primary and auxiliary modes of locomotion, which are termed motion primitives and motion transitions, respectively. Motion primitives are fundamental modes of locomotion; the five motion primitives of this study are: standing still on flat ground, walking on flat ground, walking upstairs, walking downstairs and running. Due to the differences of modeling, we separate the five motion primitives into two groups: the first group only contains a standing still mode and is termed as "stationary motion primitive"; the second group includes the remaining four locomotion behaviors and is termed the "mobile motion primitives". 
To switch between different motion primitives in a stable manner, auxiliary modes, termed "motion transitions", are introduced.

To summarize, this thesis presents two main contributions; the first is an extension of [2], in which the author presents a method of automatically obtaining robotic walking controllers, via an optimization, from a set of human walking data. In this thesis, it is shown that an augmentation of the optimization can be successfully applied to multiple modes of locomotion. An extending result of [3] is given in which it is shown that the solution to the optimization problem, which minimizes the cost of the least square fits of the control outputs to corresponding human data, ensures partial hybrid zero dynamics; and thus, yields stable walking controllers for walking on flat ground, upstairs, downstairs and running. The second contribution is that by utilizing the same optimization problem, subject to different constraints, we can achieve controllers which evolve the robot from one locomotion mode to another. The combination of these two results is a collection of controllers, automatically obtained from optimizations about human data, which form a continuous, multi-model system. 


\section{HUMAN LOCOMOTION DATA}

The most prevalent source of information on bipedal robotic walking found in nature is the human body. This chapter provides an overview of the analysis and insight obtained through examination of human locomotion. The human locomotion data considered in this work is obtained from a data set that is made public by the motion capture lab in UC Berkeley. In particular, this data set includes four types of locomotion: walking on flat ground, ascending a stairway, descending a stairway and running. Single step data is isolated from the experiment data via a procedure termed the domain breakdown for each type of motion of each subject. The extended canonical function is proposed and shown to universally represent specific kinematic outputs of the human body for all four mobile motion primitives and four motions transitions considered in this thesis.

\subsection{Human Locomotion Data}

The available on-line data set contains total of four experiments (including level walking, stair ascending, stair descending and running), which were performed at different times. According to the data explanation, all experiments are carried out by the motion capture lab in the UC Berkeley with the IRB Protocol \#2011-04-3088. For each subject, data which contain the least noise were used in the computation

of kinematic outputs. Here, the "noisy" data are mainly because of the from time to time disfunction of the sensors. An average is computed of all the subjects' data for a given output; this average is termed the "mean human data" corresponding to the kinematic output. It is the analysis of this mean human data that forms the basis of our locomotion controller design. 


\subsection{Automated Domain Breakdown}

The process by which the start and end of a single step are determined, is termed the domain breakdown procedure. Generally, the domain breakdown is obtained through a position threshold which specifies when the heel or toe is on the ground. Differently, a new method by utilizing the acceleration data-rather than positionof the heel and toe to determine the single step interval is proposed in this thesis. This method is motivated by noting that the time when the contact points (heel or toe) hit or lift the ground is the moment that maximum acceleration occurs. Data of the heel position and acceleration of a test subject during the stair ascending are shown in Fig. 2.1. The peak accelerations are indicated with dashed vertical lines. The corresponding positions of the heel show the moment of heel strike and lift.

This method is similar to the analysis of ground reaction forces using force plate, which is common in the bio-mechanics community [11, 23]. The domain breakdown plays a critical role in the human-inspired locomotion controller design process; that is, the complete-step time intervals obtained through the domain breakdown specify the intervals of data over which our function fitting method is valid, i.e., the nominal operation region of our controller.

\subsection{Extended Canonical Human Function}

In [30], it is shown that certain kinematic outputs of human walking in level ground can be represented by a single, universal function termed the "canonical human function (CHF)" - which has the similar form of the solution to a linear spring-damper system under constant force. The same function is also able to fit the outputs of human running for both stance phase and flight phase exceptionally well [39]. Examination of the data for walking up and down stairs, however, reveals a need for the augmentation of this function. It is found that walking up and down 


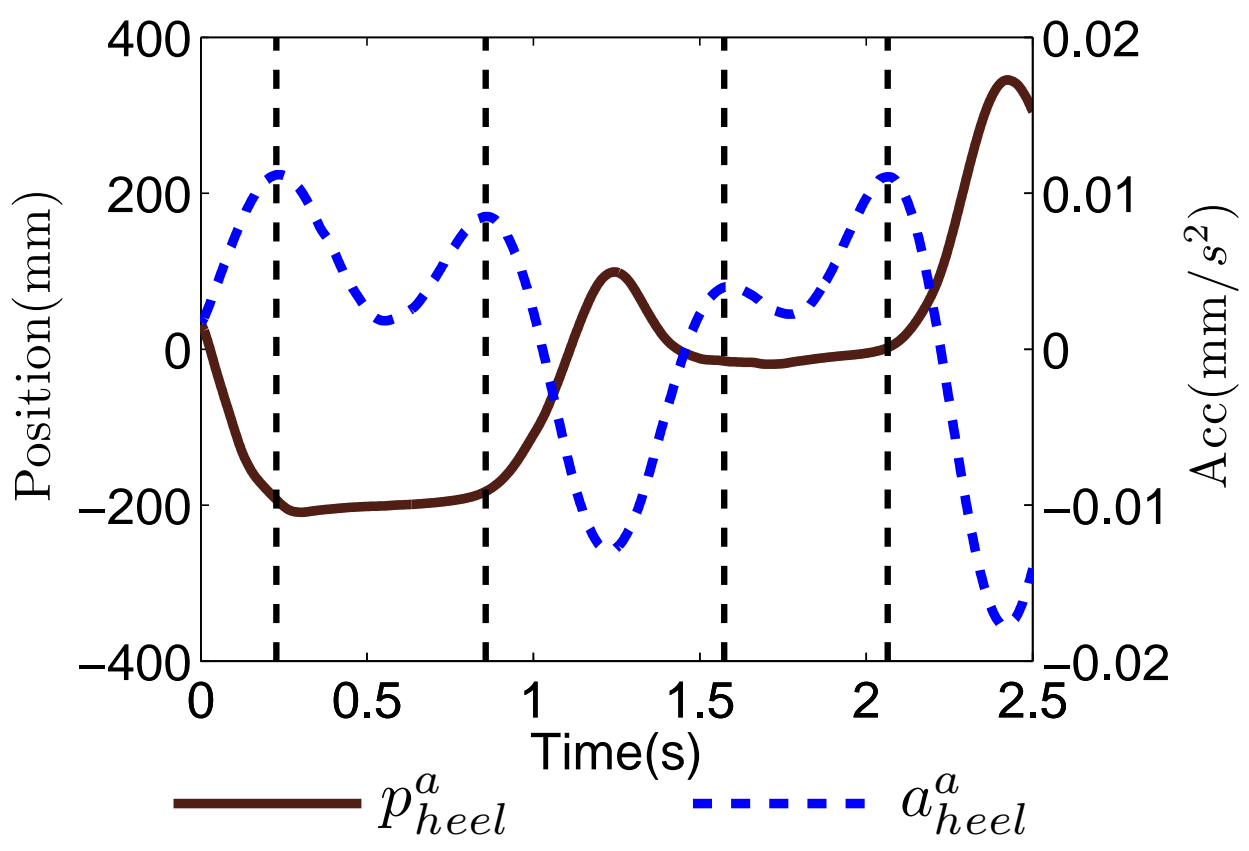

Figure 2.1: Domain breakdown for walking up stairs [38].

stairs, and the transitions between these behaviors and flat ground walking, can be represented — with a high degree of accuracy — by the response of a linear springmass-damper system subject to a sinusoidal excitation, which has the form as follows:

$$
\begin{aligned}
y=\underbrace{e^{-\zeta \omega_{n} t}\left(c_{0} \cos \left(\omega_{d} t\right)+c_{1} \sin \left(\omega_{d} t\right)\right)+g_{0} / \omega_{n}^{2}}_{\text {"human"=linear spring-mass-damper system }} \\
+\underbrace{c_{2}\left(\omega_{n}, \omega, \zeta, b\right) \cos (\omega t)+c_{3}\left(\omega_{n}, \omega, \zeta, b\right) \sin (\omega t)}_{\text {"enviroment"=sinusoidal excitation }},
\end{aligned}
$$

where $c_{0}$ and $c_{1}$ are the initial conditions decided by the initial position $y(0)$ and the initial velocity $\dot{y}(0) ; \xi$ is the damping ratio and $\omega_{n}$ is the natural frequency; $\omega_{d}=\sqrt{1-\xi^{2}} \omega_{n}$ is the damped frequency; the constant term $g_{0}$ is the gravity term; $b$ and $\omega$ are the amplitude and frequency of the sinusoidal excitation, respectively; 
$c_{2}$ and $c_{3}$ are functions of $\omega_{n}, \omega, \xi$ and $b$ given by:

$$
\begin{aligned}
& c_{2}=(2 b \xi \omega) /\left(\omega^{4}+2\left(-1+2 \xi^{2}\right) \omega^{2} \omega_{n}^{2}+\omega_{n}^{4}\right), \\
& c_{3}=\left(b\left(-\omega^{2}+\omega_{n}^{2}\right)\right) /\left(\omega^{4}+2\left(-1+2 \xi^{2}\right) \omega^{2} \omega_{n}^{2}+\omega_{n}^{4}\right) .
\end{aligned}
$$

Manipulation of (2.1) yields the following simplified form, which we term the extended canonical human function (ECHF):

$$
y_{H}(t)=e^{-\alpha_{1} t}\left(\alpha_{2} \cos \left(\alpha_{3} t\right)+\alpha_{4} \sin \left(\alpha_{3} t\right)\right)+\alpha_{5} \cos \left(\alpha_{6} t\right)+\kappa(\alpha) \sin \left(\alpha_{6} t\right)+\alpha_{7},
$$

where $\kappa(\alpha)=\left(2 \alpha_{1} \alpha_{5} \alpha_{6}\right) /\left(\alpha_{1}^{2}+\alpha_{3}^{2}-\alpha_{6}^{2}\right)$. Comparing this form with the solution of linear spring-mass-damper systems, we have $\alpha_{1}=\xi \omega_{n}, \alpha_{2}=c_{1}, \alpha_{3}=\omega_{d}, \alpha_{4}=c_{1}$, $\alpha_{5}=c_{2}, \alpha_{6}=\omega$ and $\alpha_{7}=g_{0} / \omega_{n}^{2}$. Note the form of equation (2.1), the homogenous solution terms and the gravity related constant correspond to the basic solution of the "linear spring-mass-damper" system of human locomotion while the sinusoidal excitation terms are due to the external excitation imparted on the system by the environment - which is, in this case, the elevation of the walking surface. Moreover, for the cases of level walking and running, the sinusoidal excitation terms can be removed, which is the case discussed in [2]. The reason to execute this manipulation (2.4) is that the ECHF has a simpler form that yields efficiency for the optimization problem which will be discussed later.

With the ECHF in hand, it is important to note how the ideas considered in this work, and the control approach taken, draw motivation from human walking. The realization of physical human walking is achieved via a highly sophisticated and complex system - the coordination of the muscular and nervous systems required even for the simple task of taking one step is still not fully understood. Therefore, 


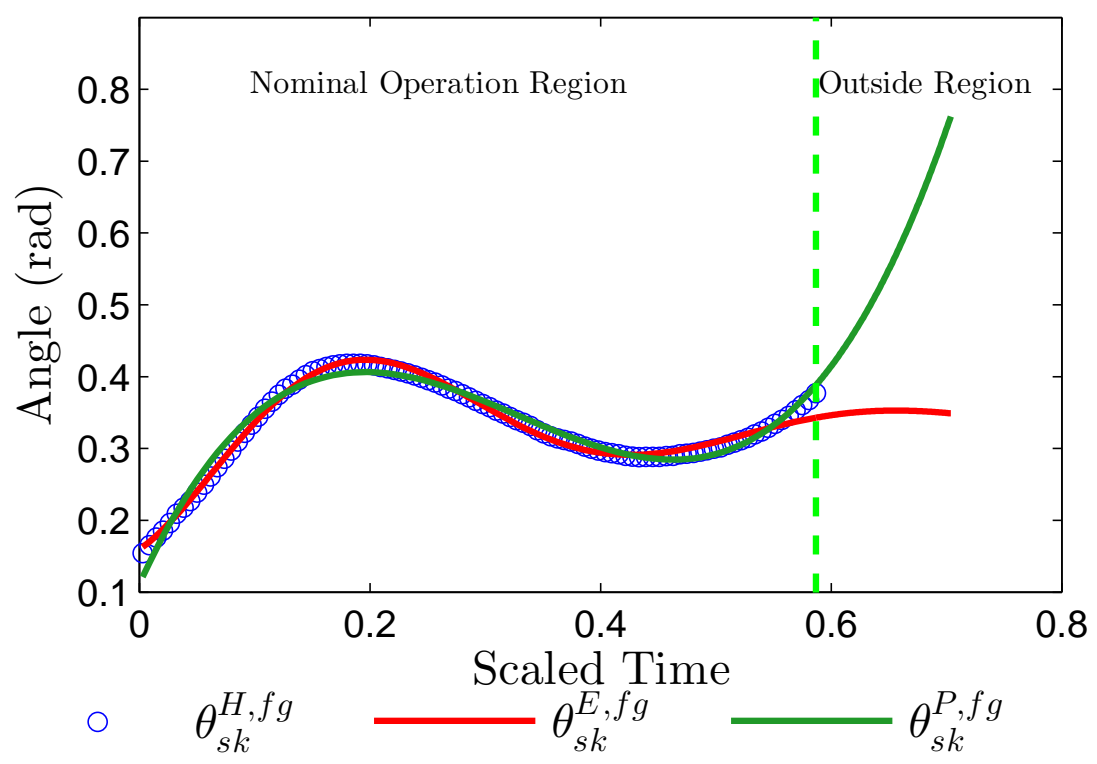

Figure 2.2: Stance knee angle data fit comparison between the ECHF and the 4th order polynomial function at the outside of the nominal operation region, which is obtained by extending $20 \%$ of the nominal operation region. The superscripts $H, E$, $P$ denote the human data, ECHF fitting and polynomial fitting, respectively [38].

rather than trying to understand the complete dynamics of human walking, we take a control theorist perspective: we view the human walking system as a "black box". In this light, the problem becomes to find the "outputs" of this black box that characterize the behavior of the system. By tracking these outputs in a robot, through virtual output constraints, the result is a robot which displays the same qualitative behaviors as the human despite the differences in dynamics. Similar approaches have been taken in the robotics community; some of these approaches to note are: polynomial functions used in [20], B-spline functions in [36] and Bezier series applied in $[32,34]$. However, unlike all these functions, the ECHF provides a simpler form (requires fewer parameters) that appears to characterize all the basic human locomotion primitives (walking, stair ascending, stair descending and running [39]). Moreover, 
another advantage to our approach over other similar methods (for example, a 4th order polynomial function used in [8]) is the better behavior of the ECHF outside the nominal operation region. As shown in Fig. 2.2, both the ECHF (with correlation as 0.9934 ) and the $4 t h$ order polynomial function (with the correlation as 0.9875 ) can fit the human data very well. However, the $4 t h$ order polynomial function blows up outside the operation window immediately and becomes infeasible while the ECHF still remains a reasonable value, which we claim is very important to give the robot more robustness while handling the external disturbance. As robot will always miss the designed right time frame in real world due to the environment disturbance and model uncertainty, the behavior outside the nominal region plays a key role to the stability and robustness of the robot. Evidence of the robustness of using the ECHF on a physical robot can be seen on ABMER [19]. In the proceeding subsection, we discuss the specific outputs chosen to characterize human walking and show that the ECHF represents these certain outputs of human data with high correlations.

\subsection{Human Outputs}

While analyzing the human data, we seek human outputs which satisfy the specific criteria, i.e., they (1) are functions of the joint angles, (2) have simple time-based representation, and (3) are mutually exclusive (the decoupling matrix associated with these outputs is non-singular). A total of four kinematic outputs are required for the fully actuated 4-DOF robot model in consideration. Analysis of the human data yields the following four outputs (seen in Fig. 3.1c) which seem to fully describe the 4-DOF analog of the human locomotion system:

1. the linearized forward position of the hip,

$$
\delta p_{h i p}=-\theta_{s f} L_{c}+\left(-\theta_{s f}-\theta_{s k}\right) L_{t}
$$


where $L_{c}$ and $L_{t}$ are the lengths of the calf and thigh, respectively; and $\theta_{s f}$ is stance ankle angle measured from the vertical line to the calf, which can be seen in Fig. 3.1a,

2. the hip angle, $\theta_{\text {hip }}$, which is the angle measured from stance thigh to non-stance thigh;

3. the stance knee angle $\theta_{s k}$;

4. the non-stance knee angle, $\theta_{n s k}$.

To calculate the mean human outputs, the following three steps are performed. First, we compute the kinematic outputs, from human data, of all six subjects considered in this thesis. Second, we determine which subject has the shortest single step interval, via using the domain breakdown procedure from Sect. 2.2. Then we denote this step interval the "reference step interval". For all other subjects, we scale all four kinematic outputs to the reference step interval. Finally, we average the outputs of all six subjects to obtain the mean human outputs, which can be seen in Fig. 2.3 and Fig. 2.4. Explicitly, $f g$, us, $d s$ correspond to the data of walking on flat ground, upstairs and downstairs, respectively. And $f \rightarrow u$ represents the data of transition from walking on flat ground to walking on upstairs; similar definition holds for other similar terms.

Examination of human locomotion data reveals that the time-derivative of linearized hip position is approximately constant, as seen in Fig. 2.3a and Fig. 2.4a. Thus, we fit the linearized hip position with straight line as $\delta p_{h i p}^{d}=\mathrm{v}_{\text {hip }} t$, where $\mathrm{v}_{h i p}$ is the forward hip velocity. Utilizing the ECHF, the remaining three desired outputs 


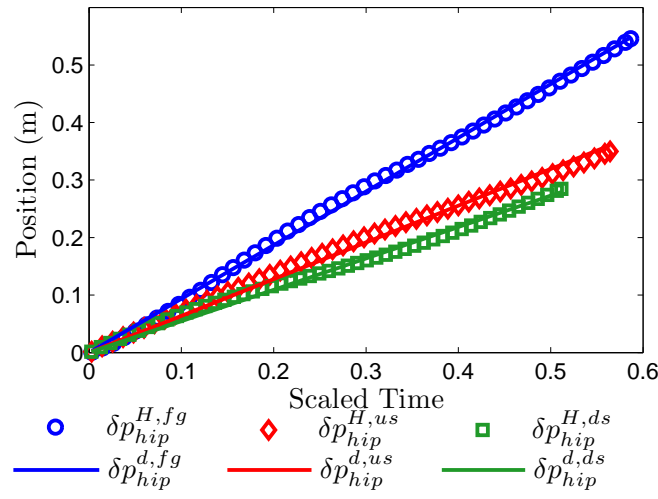

(a) Fitted hip position

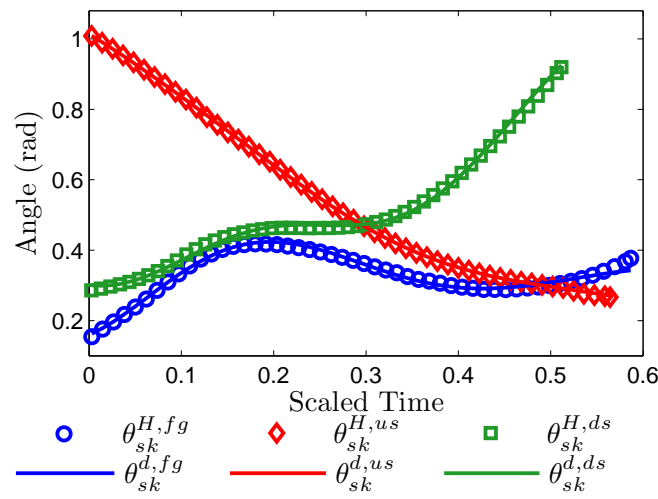

(c) Fitted stance knee angle

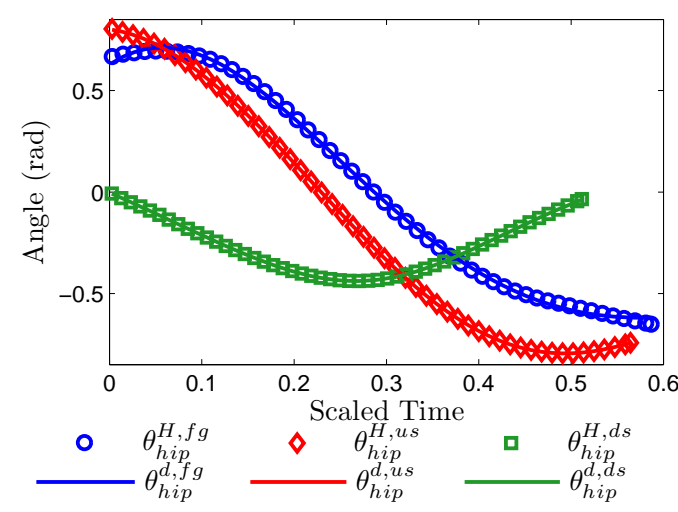

(b) Fitted nonstance hip angle

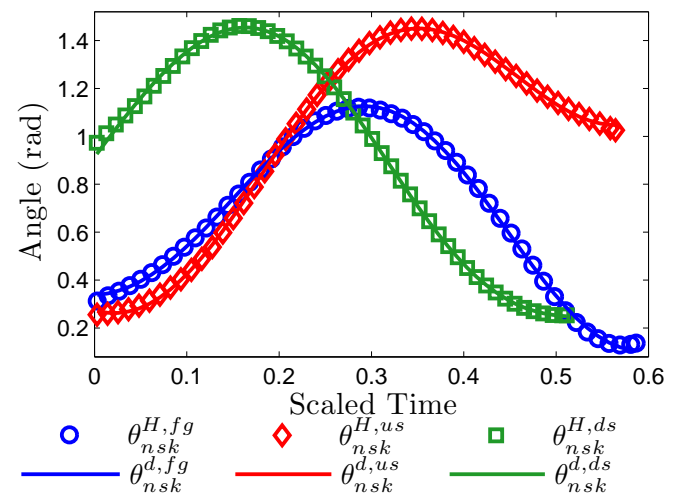

(d) Fitted nonstance knee angle

Figure 2.3: Fitted ECHF and corresponding mean human data of motion primitives [38].

of robot can be stated as:

$$
\begin{aligned}
& \theta_{h i p}^{d}\left(t, \alpha_{h i p}\right)=y_{H}\left(t, \alpha_{h i p}\right), \\
& \theta_{s k}^{d}\left(t, \alpha_{s k}\right)=y_{H}\left(t, \alpha_{s k}\right), \\
& \theta_{n s k}^{d}\left(t, \alpha_{n s k}\right)=y_{H}\left(t, \alpha_{n s k}\right),
\end{aligned}
$$

where, e.g., $\alpha_{s k}=\left(\alpha_{s k, 1}, \alpha_{s k, 2}, \alpha_{s k, 3}, \alpha_{s k, 4}, \alpha_{s k, 5}, \alpha_{s k, 6}, \alpha_{s k, 7}\right)$ in equation (2.4). The parameters of all the outputs can be combined into a single parameter vector: $\alpha=$ $\left(\mathrm{v}_{\text {hip }}, \alpha_{\text {hip }}, \alpha_{s k}, \alpha_{n s k}\right) \in \mathbb{R}^{22}$. By fitting these functions, via least square fits which 


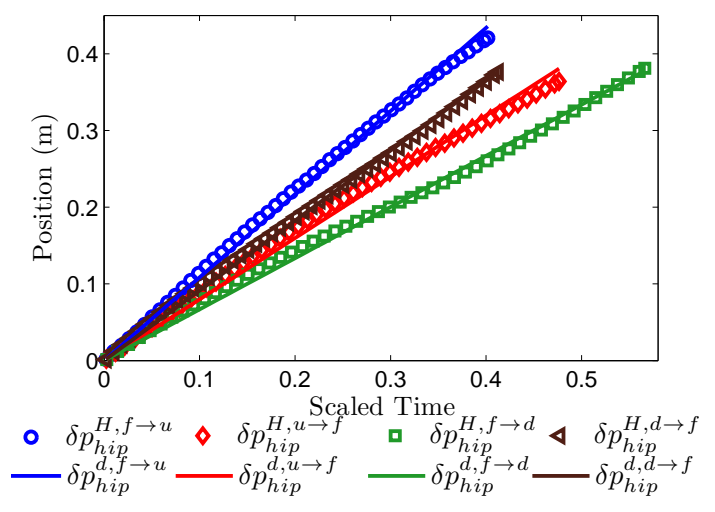

(a) Fitted hip position

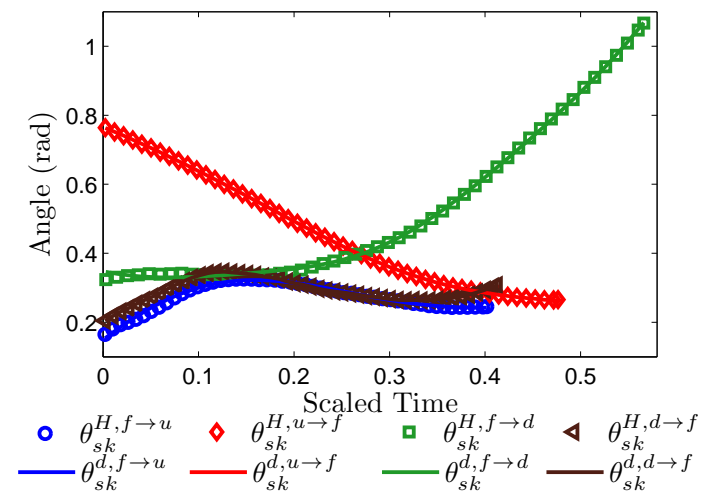

(c) Fitted stance knee angle
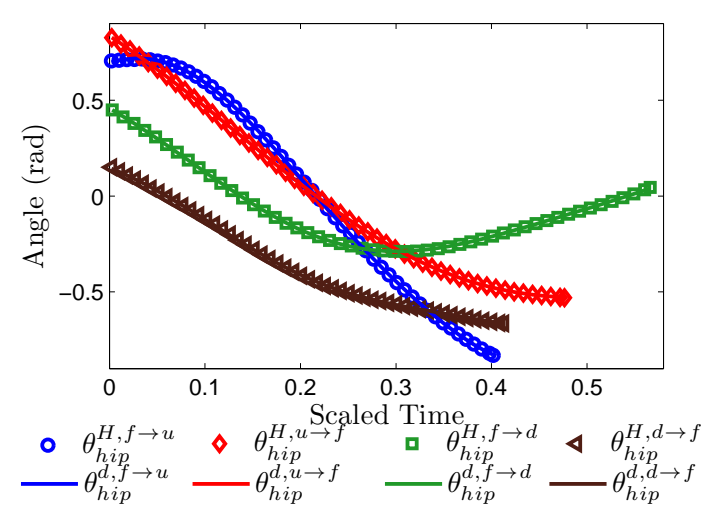

(b) Fitted nonstance hip angle

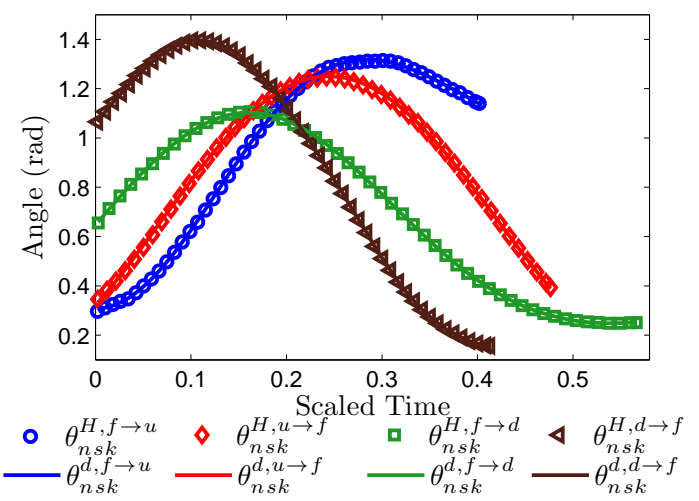

(d) Fitted nonstance knee angle

Figure 2.4: Fitted ECHF and corresponding mean human data of motion transitions [38].

yield high correlation coefficients, to corresponding human data, we claim that the canonical human function accurately describes human walking data.

To determine the parameters for the ECHF, the following optimization is solved:

$$
\alpha^{*}=\underset{\alpha \in \mathbb{R}^{22}}{\operatorname{argmin}} \operatorname{Cost}_{\mathrm{HD}}(\alpha)
$$

This optimization problem produces the least square fits (the detailed form of the cost function can be referred to [2]) of the ECHF to the corresponding human data. The parameters obtained through this process are given in Table 7.1, together with the 
correlation of each function to the corresponding set of data. Additionally, the functions for each kinematic constraint and each locomotion behavior (three one-phase motion primitives and four motion transitions) are plotted with the corresponding human data in Fig. 2.3 and Fig. 2.4. Note that all the correlations are higher than 0.99, which implies that the ECHF can be fitted to the outputs of all three types of motion primitive and four types of motion transition universally with a high degree of accuracy.

Different for level walking and the stair climbing, the motion primitive of running includes two phases: stance phase (with one foot on the ground) and flight phase (with both feet in the air). Therefore, two CHFs are used for the fitting of running data for each phase, the results of which are shown in Fig. 2.5. Note that, as mentioned before, the CHF is a special form of ECHF with the extended term set to 0 . The fitting correlations of running data are shown in Table. 7.2, from which we can see all the correlations are greater than 0.99 . 


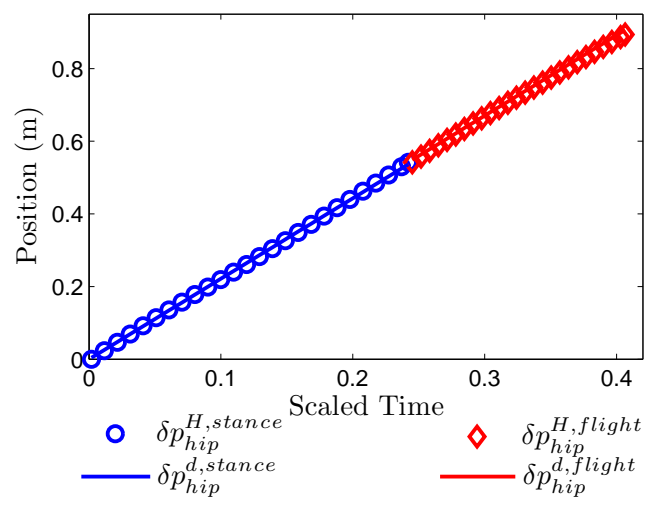

(a) Fitted hip position

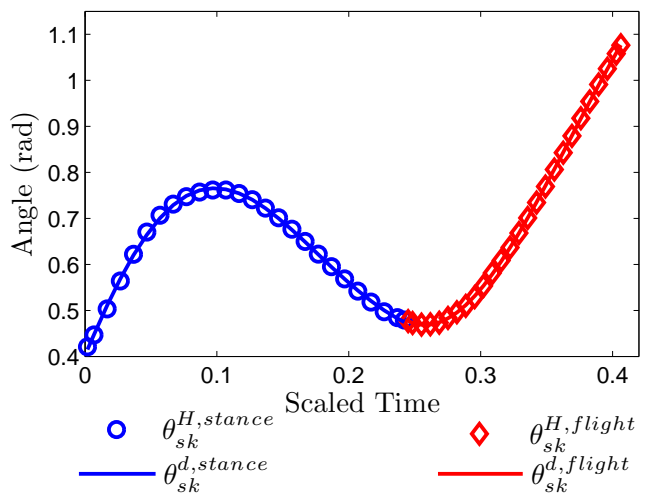

(c) Fitted stance knee angle

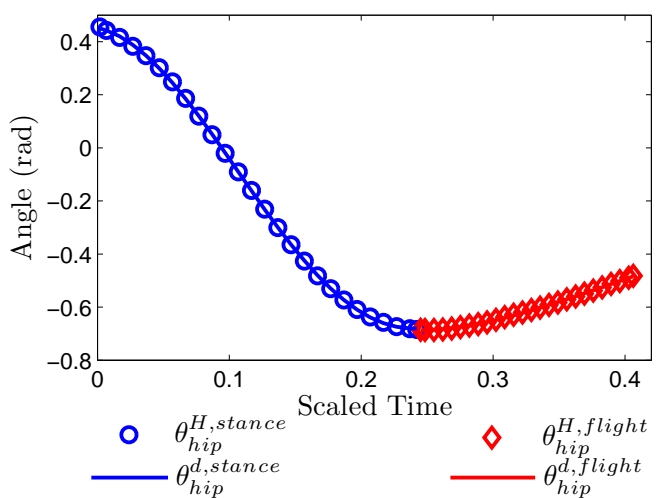

(b) Fitted nonstance hip angle

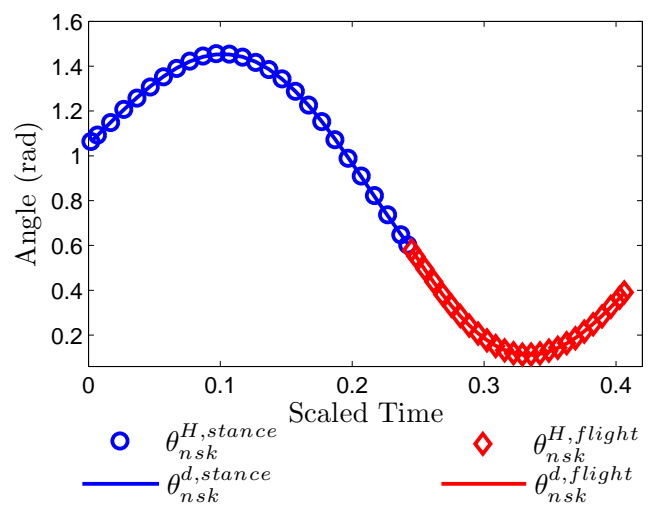

(d) Fitted nonstance knee angle

Figure 2.5: Fitted CHF and corresponding mean human data of running [39]. 


\section{ROBOT MODEL}

With the goal to achieve human-like robotic walking, a robotic model with mass and length parameters corresponding to human subject data is considered. To reduce computational complexity, while preserving the form of the human lower-body, the model is constructed as a serial chain of rigid links. Each link $l$ has a length $L_{l}$ and a mass $m_{l}$, which is a point, mass located a distance $r_{l}$ from the base of the link. The resulting model configuration is shown in Fig. 3.1a, while the mass and length distribution are shown in Fig. 3.1b. The specific values of these parameters for each

single subject are obtained by applying Winter's [35] mass and length distribution to the human test subject. The mass and length of all subjects considered in this thesis are averaged to obtain the parameters of mean human data model. The modeling process considers the common assumptions in the bipedal robot literature, which imply the existence of both continuous and discrete dynamic behavior that observed in phases of single support and double support. Based on the model specifications, the following sections give the derivation of the dynamics of the system.

\subsection{Continuous Dynamics}

Given the configuration space $\mathcal{Q}: q=\left\{\theta_{s f} ; \theta_{s k} ; \theta_{h i p} ; \theta_{n s k}\right\}$, using the method of Lagrangian with the consideration of holonomic constraints, we can obtain the dynamics of the continuous phase as:

$$
D(q) \ddot{q}+H(q, \dot{q})+A^{T}(q) \lambda=B(q) u
$$

where, $D(q)$ and $B(q)$ are the generalized inertia and torque distribution maps, respectively, and $H(q, \dot{q})=C(q, \dot{q}) \dot{q}+G(q)$ contains terms resulting from the Coriolis 


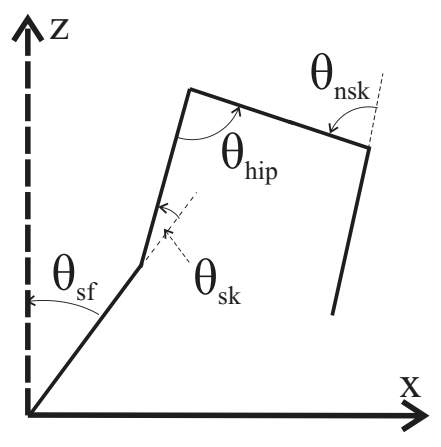

(a) Robot configuration

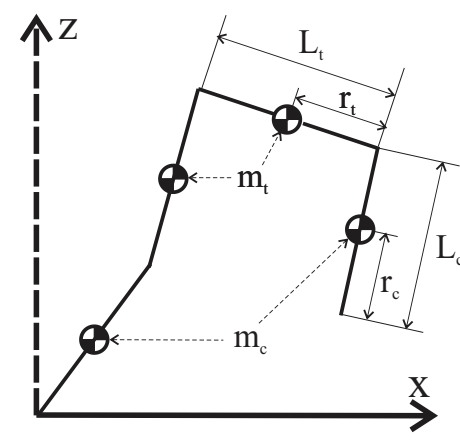

(b) Robot mass-length

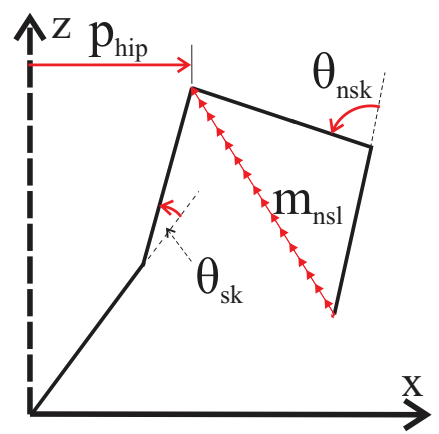

(c) Robot outputs

Figure 3.1: The modeled robot's configuration, mass and length distribution, and virtual constraints [38].

effect, centrifugal forces and gravity. Note that, here $B(q) u$ provides full actuation as modeled, which will be discussed later in Sect. 4. $A(q) \in \mathbb{R}^{k \times n}$ corresponds to a set of $k$ holonomic constraints; $\lambda$ are Lagrange Multipliers which are used to enforce holonomic constraints. The formula of $\lambda$ can be given with the method from Chapter 6 in $[26]$ as:

$$
\lambda=\left(A(q) D(q)^{-1} A(q)^{T}\right)^{-1}\left(\dot{A}(q) \dot{q}+A(q) D^{-1}(B(q) u-H(q, \dot{q}))\right) .
$$

Substituting this equation back into equation (3.1) leads to the affine control system for the continuous dynamics, $F G$ :

$$
\dot{x}=f(q, \dot{q})+g(q) u,
$$


where $f(q, \dot{q})$ and $g(q)$ are defined as:

$$
\begin{gathered}
f(q, \dot{q})=\left[\begin{array}{c}
\dot{q} \\
D^{-1}(q)\left(A^{T}(q) \Theta(q) A(q) D^{-1}(q)-I\right) \\
\left(H(q, \dot{q})-A^{T}(q) \Theta(q) \dot{A}(q) \dot{q}\right)
\end{array}\right], \\
g(q)=\left[\begin{array}{c}
0 \\
D^{-1}(q)\left(I-A^{T}(q) \Theta(q) A(q)\right) B(q)
\end{array}\right],
\end{gathered}
$$

where, for simplicity $\Theta(q)=A(q) D^{-1}(q) A^{T}(q)$.

Particularly, two types of continuous walking behavior with different dynamic models are considered. The first type is stationary behavior that contains standing still on flat ground, in which case both feet are pinned to the ground. The second type is a set of four mobile behaviors, in which case maximum one leg is pinned to the ground while the other leg swings freely. In particular, we define the two types of motion primitives as follows:

Stationary Motion Primitive. For stationary behavior, Lagrange Multipliers are needed to ensure that the swing foot remains in contact with the ground. Thus, $f_{s}(q, \dot{q})$ and $g_{s}(q)$ are defined with the form given by (3.5).

Mobile Motion Primitive. For the mobile behavior, the swing foot is not constrained, i.e., $A(q)=0$. Thus, the affine control system of mobile locomotion can be reduced to the following form:

$$
f_{m}(q, \dot{q})=\left[\begin{array}{c}
\dot{q} \\
D^{-1}(q) H(q, \dot{q})
\end{array}\right], \quad g_{m}(q)=\left[\begin{array}{c}
\mathbf{0} \\
D^{-1}(q) B(q)
\end{array}\right]
$$




\subsection{Discrete Dynamics}

With the modeling assumption that the discrete dynamics phase occurs instantaneously, the dynamic response is modeled as an impact on the system. Specifically, the method of [17] is used to model the plastic rigid-body impacts as impulse responses. The detailed derivation can be found in Chapter 3 in [33], thus, is omitted here for space consideration.

With the assumption of symmetric walking, we use a stance/non-stance notation for the legs. To affect this statement that the legs be "switched" at impact, and thereby reduce the complexity of the model, the reset map can be implemented as following:

$$
\Delta(q, \dot{q})=\left[\begin{array}{c}
\Delta_{q} q \\
\Delta_{\dot{\theta}}(q) \dot{\theta}
\end{array}\right]
$$

where $\Delta_{q}$ is the relabeling $[2,13]$ which switches the stance and non-stance leg at impact; and $\Delta_{\dot{\theta}}$ determines the change of velocity due to the impact. Further details can be found in $[2]$. 


\section{CONTROLLER DESIGN}

The purpose of this chapter is to specify a controller, $u$, for the given control system (3.5). Motivated by the desire to obtain human-like bipedal robotic locomotion, we seek to construct a controller which drives outputs of the robot to corresponding outputs of the human. Formally, we seek a $u_{\alpha}$ which guarantees that $y^{a}(q) \rightarrow y_{\alpha}^{d}(t)$ as $t \rightarrow \infty$, where $y^{a}$ are the outputs of the robot (computed via kinematics) and $y_{\alpha}^{d}$ are the outputs of the human as represented by the ECHF. As the dynamics of the robot model are highly nonlinear, the natural choice of control method for this system is Feedback Linearization [29].

\subsection{Parameterization of Time}

Autonomous control has several advantages for the control of bipedal robots (see [4]). With this consideration, we introduce a state-based parameterization of time in our system; this is a common practice in $[33,34]$. As shown in Section 2.4, analysis of human data reveals that the linearized forward position of the hip $\delta p_{\text {hip }}$ evolves in an approximately-linear manner with respect to time, that is $\delta p_{\text {hip }}\left(t, \mathrm{v}_{\text {hip }}\right) \approx \mathrm{v}_{\text {hip }} t$. Taking advantage of this observation, the following parameterization of time is formed:

$$
\tau(q)=\frac{\delta p_{\text {hip }}^{R}(q)-\delta p_{\text {hip }}^{R}\left(q^{+}\right)}{v_{h i p}}
$$

where $\delta p_{\text {hip }}^{R}\left(q^{+}\right)$is the linearized forward position of the hip at the beginning of the current step.

\subsection{Controller Specification}

With the parameterization of time in place, the control law can be defined explicitly with the ECHF discussed in Sect. 2. Particularly, we define the (relative degree 
2) actual outputs of the robot to be the output functions considered in Sect. 2 and the desired outputs to be the outputs of the human as represented by the ECHF but with parameterized time $\tau(q)$ :

$$
y_{2}^{a}(\theta)=\left[\begin{array}{c}
\theta_{h i p} \\
\theta_{s k} \\
\theta_{n s k}
\end{array}\right], y_{2, \alpha}^{d}(\theta)=\left[\begin{array}{c}
\theta_{h i p}^{d}\left(\tau(q), \alpha_{h i p}\right) \\
\theta_{s k}^{d}\left(\tau(q), \alpha_{s k}\right) \\
\theta_{n s k}^{d}\left(\tau(q), \alpha_{n s k}\right)
\end{array}\right] \text {. }
$$

Similarly, with the goal of controlling the velocity of the robot, we define the relative degree 1 outputs to be the velocity of the hip and the desired velocity of the hip:

$$
y_{1}^{a}(\theta, \dot{\theta})=d \delta p_{\text {hip }}(\theta) \dot{\theta}, \quad y_{1, \alpha}^{d}=\mathrm{v}_{\text {hip }} .
$$

The goal is for the outputs of the robot to agree with the outputs of the human, therefore, motivating the final form of the human-inspired outputs to be used in feedback linearization:

$$
y_{\alpha}(q, \dot{q})=\left[\begin{array}{c}
y_{1, \alpha}(q, \dot{q}) \\
y_{2, \alpha}(q)
\end{array}\right]=\left[\begin{array}{c}
y_{1}^{a}(\theta, \dot{\theta})-\mathrm{v}_{h i p} \\
y_{2}^{a}(q)-y_{2, \alpha}^{d}(q)
\end{array}\right] .
$$

Therefore, the feedback linearization controller, $u_{\alpha}(q, \dot{q})$, can now be stated as:

$$
\begin{aligned}
u_{\alpha}(q, \dot{q})= & -\mathcal{A}_{\alpha}^{-1}(q, \dot{q})\left(\left[\begin{array}{c}
0 \\
L_{f} L_{f} y_{2, \alpha}(q, \dot{q})
\end{array}\right]\right. \\
& \left.+\left[\begin{array}{c}
L_{f} y_{1, \alpha}(q, \dot{q}) \\
2 \varepsilon L_{f} y_{2, \alpha}(q, \dot{q})
\end{array}\right]+\left[\begin{array}{c}
\varepsilon y_{1, \alpha}(q, \dot{q}) \\
\varepsilon^{2} y_{2, \alpha}(q)
\end{array}\right]\right),
\end{aligned}
$$


with control gain $\varepsilon$ and decoupling matrix $\mathcal{A}_{\alpha}(q, \dot{q})$ given by

$$
\mathcal{A}_{\alpha}(q, \dot{q})=\left[\begin{array}{c}
L_{g} y_{1, \alpha}(q, \dot{q}) \\
L_{g} L_{f} y_{2, \alpha}(q, \dot{q})
\end{array}\right] .
$$

Due to one of the criteria that the outputs should be mutually exclusive [2], the decoupling matrix is guaranteed to be non-singular. It follows that for a control gain $\varepsilon>0$, the control law $u_{\alpha}$ renders the outputs exponentially stable [29]. That is, the outputs of the robot converge to the ECHF exponentially. Note that, since the parameters $\alpha$ are so important and change for each motion primitive, we explicitly define the controller $u_{\alpha}$ and desired output functions $y_{\alpha}^{d}$ corresponding to $\alpha$. The goal of Chapter 6 will be to determine the parameters, $\alpha$, of this control law to achieve different walking behaviors based upon the human data. 


\section{HYBRID AND META-HYBRID SYSTEMS}

In this chapter, it is shown that primary modes of bipedal locomotion-such as walking, standing, traversing stairways and running - can each be represented by a unique hybrid control system. However, control of functional bipedal robots requires dominion over multiple primary modes of locomotion. Therefore, to develop a functional locomotion control scheme, one must introduce auxiliary hybrid systems, which evolve the state of the robot during transitions between primary modes. To this end, we propose the concept of a meta-hybrid system, which consists of both primary and auxiliary hybrid systems formally merged together in a way that allows for the motion planning through both motion primitives and motion transitions.

\subsection{Hybrid System for the Biped}

Given the Lagrangian and impact dynamics of the robot model in Section 3, a natural choice of mathematical representation for this model is a hybrid system [2], which exhibits both continuous and discrete dynamics. Formally, we begin by writing the hybrid control system for the robot as:

$$
\mathscr{H} \mathscr{C}_{h}^{R}=\left(\mathcal{D}_{h}^{R}, \S_{h}^{R}, \Delta^{R}, f^{R}, g^{R}, U^{R}\right),
$$

which depends on a unilateral constraint function, $h$, representing the terrain of the hybrid system. The superscript $R$ denotes that the notations following are for the robot in this thesis particularly. Specifically, $h$ is the height of non-stance foot above the walking surface, e.g. a staircase or level ground; $h$ characterizes the allowable configuration, i.e., the domain. With both feet on flat ground, the stationary 
behavior domain is defined as:

$$
\mathcal{D}_{h_{s}}^{R}=\left\{(q, \dot{q}) \in T Q: h_{s}(q)=0 \text { and } \frac{\partial h_{s}(q)}{\partial q} \dot{q}=0\right\}
$$

Similarly, the domain of mobile behaviors can be stated as:

$$
\mathcal{D}_{h_{m}}^{R}=\left\{(q, \dot{q}) \in T Q: h_{m}(q) \geq 0\right\}
$$

The guard is the boundary of the domain with the additional assumption that the unilateral constraint is decreasing, as stated as following:

$$
\S_{h}^{R}=\left\{(q, \dot{q}) \in T Q: h(q)=0 \text { and } \frac{\partial h(q)}{\partial q} \dot{q}<0\right\} .
$$

The remaining elements are specified by the dynamics of the robot; that is, they are intrinsic to the model and consistent for all hybrid system representations of the robot, yet they are independent of the terrain. These elements are given by:

- $\Delta^{R}$ is the reset map, corresponding to the impact equations as defined in (3.7),

- $U^{R}=\mathbb{R}^{4}$, as we assume full control authority.

Applying the human-inspired feedback control law $u(\theta, \dot{\theta})$ as defined in $(4.5)$, we have the hybrid system as:

$$
\mathscr{H}_{(h, \alpha)}^{R}=\left(\mathcal{D}_{h}^{R}, \S_{h}^{R}, \Delta^{R}, f_{\alpha}^{R}\right)
$$

with $f_{\alpha}^{R}(\theta, \dot{\theta})=f^{R}(\theta, \dot{\theta})+g^{R}(\theta) u_{\alpha}(\theta, \dot{\theta})$.

Note that, as stated in Sect. 3.1, we have different $\left(f^{R}, g^{R}\right)$ with respect to the domains, i.e., the types of motion primitive. Therefore, with different domains and 
$\left(f^{R}, g^{R}\right)$, we define the different types of hybrid control system as $\mathscr{H}_{(h, \alpha)}^{R, i}$ with $i=s$ or $m$ corresponding to the stationary motion primitive and mobile motion primitives, respectively.

For each type of hybrid system, we have made the dependence of $f_{\alpha}^{R}$ on the parameters $\alpha \in \mathbb{R}^{22}$ of the human walking functions explicitly (note that $f_{\alpha}^{R}$ also depends on the control gain $\varepsilon$, but since the same gain will be used in all cases for the robot, it is not explicitly stated). The end result of the modeling process is that two different types of hybrid system are defined based on the types of motion primitive. Specifically, for each type of motion, a hybrid system $\mathscr{H}_{(h, \alpha)}^{R, i}$ that depends on the type of behavior (through $i=s$ or $m$ ), the terrain it is walking in (through $h$ ) and the parameters of the human inspired control $\alpha$, has been defined.

Note that, considering the running motion primitive consists two phases (stance and flight), the general form of domain as discussed above contains two sub-domains, i.e., stance sub-domain and flight sub-domain. In particular, because of the transition between stance sub-domain to the flight sub-domain is a identity map. We still adopt the same hybrid system the definition for running. This thesis will focus on the general structure of motion primitives and motion transitions. Therefore, the detailed construction of running is omitted here, and can be referred to [39].

\subsection{Meta-Hybrid Systems}

A meta-hybrid system is a hybrid system of hybrid systems, which contains multiple locomotion behaviors and transitions between these behaviors.

Definition 1. A meta-hybrid system is a tuple,

$$
\mathscr{M} \mathscr{H}=(\Gamma, \mathscr{M}, \mathscr{T})
$$


where

- $\Gamma=(V, E)$ is a directed graph, with $V$ a set of vertices, or nodes, and $E \subset Q \times Q$ a set of edges; for $e=\left(q, q^{\prime}\right) \in E$, denote the source of $e$ by $\operatorname{sor}(e)=q$ and the target of $e$ by $\operatorname{tar}(e)=q^{\prime}$.

- $\left\{\mathscr{M}_{v}\right\}_{v \in V}$ is a collection of motion primitives, each represented by a hybrid system:

$$
\mathscr{M}_{v}=\left(\mathcal{D}_{v}, \S_{v}, \Delta_{v}, f_{v}\right)
$$

- $\left\{\mathscr{T}_{e}\right\}_{e \in E}$ is a collection of motion transitions, represented by hybrid systems of the form:

$$
\mathscr{T}_{e}=\left(\mathcal{D}_{\operatorname{tar}(e)}, \S_{\operatorname{tar}(e)}, \Delta_{\operatorname{tar}(e)}, f_{e}\right),
$$

That is, $\mathscr{T}_{e}$ has the same domain, guard and reset map as $\mathscr{M}_{\operatorname{tar}(e)}$, but has a different vector field $f_{e}$.

Hybrid Period Orbits and the Poincaré Map. In order to establish the stability of k-periodic orbits, we will use the standard technique of studying the corresponding Poincaré map. In particular, taking $G$ to be the Poincaré section, one obtains the Poincaré map, $P: G \rightarrow G$, which is a partial map defined by:

$$
P(z)=c(\tau(z))
$$

where $c(t)$ is the solution to $\dot{x}=f(x)$ with $c(0)=R(z)$ and $\tau(z)$ is the time-to-impact function. In particular, if $z^{*}$ is a $k$-fixed point of $P$ (under suitable assumptions on $z^{*}, G$, and the transversally of $\mathcal{O}$ and $G$ ) a $k$-periodic orbit $\mathcal{O}$ with $z^{*} \in \mathcal{O}$ is locally exponentially stable if and only if $P^{k}$ is locally exponentially stable (as a discretetime dynamical system, $z_{i+1}=P\left(z_{i}\right)$ )(the detailed definition can also be seen in 
[3]). Although it is not possible to explicitly compute the Poincaré map, one can compute a numerical approximation of this map through simulation and thereby test its stability numerically. This gives a concrete method for practically testing the stability of periodic orbits.

In this thesis, we consider a Poincaré map for hybrid systems (e.g., a meta-hybrid system) that are no longer "simple" — meaning that they exhibit multi-domain, i.e., more than one type of impact will happen. The stability of a hybrid system which undergoes sequences of different domains has been discussed in detail in [14]. Particularly, a periodic orbit can be constructed transversally; therefore, a nontrivial Poincaré map can be computed explicitly to numerically prove the stability of multi-domain hybrid system. Here, the robot will evolve through three motion primitives and two motion transitions, which will incur five impacts for one periodic orbit. Numerical approximations of the eigenvalues of the Poincaré map for the meta hybrid system are obtained by simulating the system, from one motion primitive, through a motion transition, to the next motion primitive. The meta-hybrid system is deemed stable if for every motion primitive - motion transition - motion primitive cycle, the eigenvalues have magnitude less than unity. 


\section{MOTION PRIMITIVES \& TRANSITIONS}

In this chapter, we will explicitly construct a meta-hybrid system for a bipedal robot, with the motion primitives and transitions between these behaviors. Following this construction, the execution of this meta-hybrid system will be introduced. The resulting behavior displayed by the robot, while performing these transitions and motion primitives, will be shown via results from simulation. Formally, the goal of this chapter is to construct a meta-hybrid system for the bipedal robot:

$$
\mathscr{M} \mathscr{H}^{R}=\left(\Gamma^{R}, \mathscr{M}^{R}, \mathscr{T}^{R}\right)
$$

With the four motion primitives: standing still on flat ground ss, walking on flat ground $f g$, walking up stairs $u s$, and walking down stairs $d s$, we have the directed graph $\Gamma^{R}=\left(V^{R}, E^{R}\right)$ with

$$
\begin{aligned}
V^{R} & =\{s s, f g, u s, d s, r n\} \\
E^{R} & =\{(s s, f g),(f g, s s),(f g, u s),(u s, f g),(f g, d s),(d s, f g),(f g, r n),(r n, f g)\} .
\end{aligned}
$$

Note that the transitions between standing still on flat ground to going up or down stairs and the transitions between going up stairs and going downstairs are not considered in this work for the sake of simplicity, but the methods outlined in this thesis could still be applied. The transition between standing still and running is also not considered in this work for realistic consideration (particular level walking speed need to be achieved before the running motion can be triggered). The total graph $\Gamma^{R}$ can be seen in Fig. 6.1. The remainder of this chapter will be devoted to constructing the motion primitives and motion transitions. 


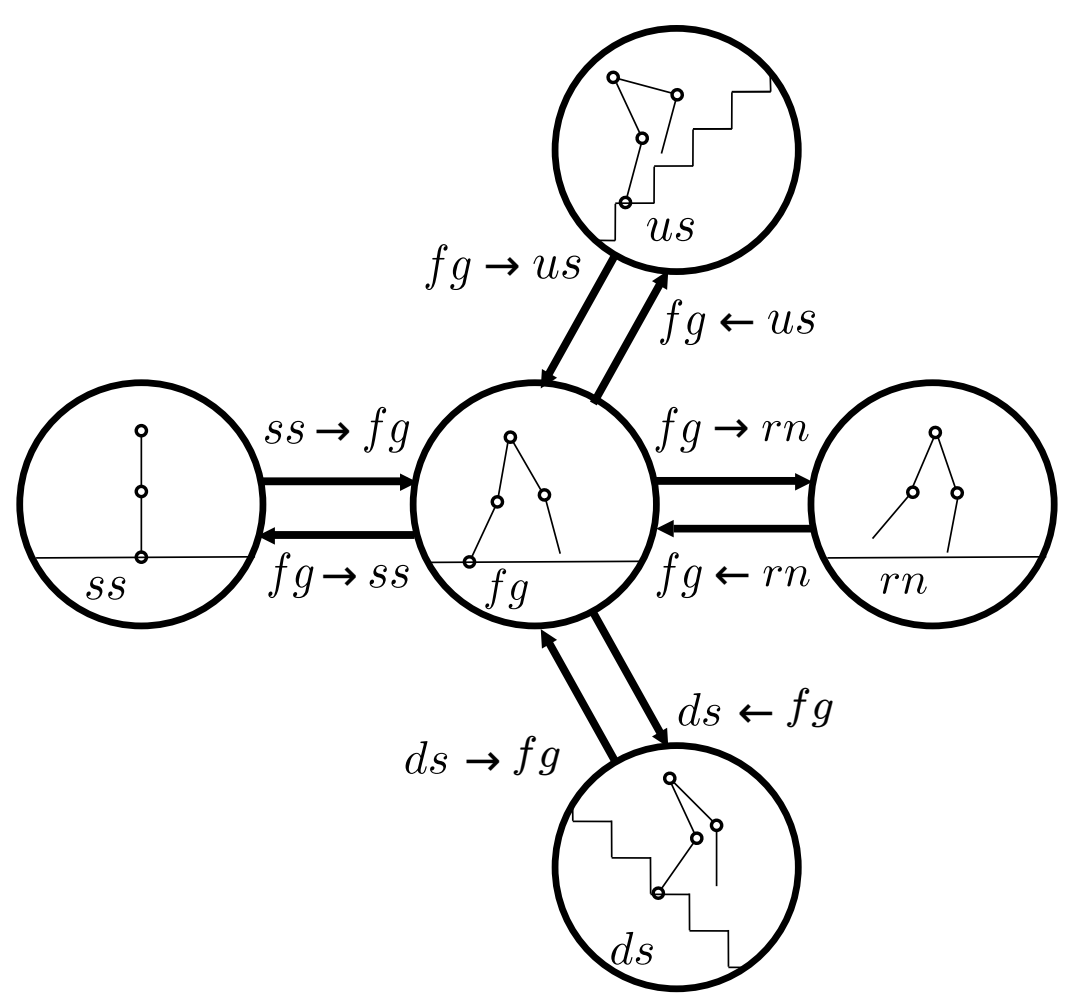

Figure 6.1: Graph of a meta-system representation for the five motion primitives in consideration.

\subsection{Motion Primitives}

Motion primitives are the core modes of locomotion of this study; this section discusses the development of controllers for motion primitives and the simulations resulting from the application of these controllers to the robot model.

\subsubsection{Motion Primitive Collection}

Using the concepts developed throughout this thesis, we can now construct mathematical representations of a bipedal robot conducting each of the four different motion primitives of interest. In particular, we can model the robot in different domains as follows:

- Standing Still on Flat Ground: $\mathscr{H}_{\left(h_{s s}, \alpha_{s s}\right)}^{R, s}$, where $h_{s s}(q)=0$, 
- Walking on Flat Ground: $\mathscr{H}_{\left(h_{f g}, \alpha_{f g}\right)}^{R, m}$, where $h_{f g}(q)=z_{n s f}(q)$,

- Walking Up Stairs: $\mathscr{H}_{\left(h_{u s}, \alpha_{u s}\right)}^{R, m}$, where $h_{u s}(q)=z_{n s f}(q)-z_{\text {stair }}$,

- Walking Down Stairs: $\mathscr{H}_{\left(h_{d s}, \alpha_{d s}\right)}^{R, m}$, where $h_{d s}(q)=z_{n s f}(q)+z_{\text {stair }}$.

- Runnig on Flat Ground: $\mathscr{H}_{\left(h_{r n}, \alpha_{r n}\right)}^{R, m}$, where $h_{r n}(q)=z_{n s f}(q)$,

The superscript $s$ of $\mathscr{H}_{\left(h_{s s}, \alpha_{s s}\right)}^{R, s}$ corresponds to the stationary motion primitive with the affine control system defined by (3.5) in which the holonomic constraints are enforced and the domain defined by (5.2). Similarly, the mobile motion primitives are denoted with superscript $m$ with the control system defined by (3.6) and the domain defined by (5.3). To effect these behaviors on the robot, it is necessary to design controllers for each motion primitive, i.e., determine the control parameters $\alpha_{v}, v \in V^{R}$, which will result in stable locomotion for the robot in each domain.

\subsubsection{Controller Development}

Because of the differences between the two groups of motion primitives, we introduce two different methods of obtaining the control parameters $\alpha_{v}, v \in V^{R}$.

\subsubsection{Stationary Motion Primitive}

For the stand still motion primitive, holonomic constraints and Lagrange Multipliers are introduced to ensure that both feet are pinned to the ground while the angles and the velocities of the system are driven to zero. A time based tracking controller, therefore, is constructed for the stationary motion primitive.

\subsubsection{Mobile Motion Primitives}

The goal of this subsection is to discuss the main contribution of this thesis; that is, starting from human data directly, through an optimization algorithm, which is subject to specific constraints, we obtain control parameters $\alpha_{v}, v \in V^{R}$ for all 
four mobile motion primitives. These control parameters guarantee stable walking while simultaneously achieving a high degree correlation fit to the human data. This methodology has been discussed in [2] for the case of flat ground walking. Here we extend the methodology to all the four mobile motion primitives. We also show that, with reasonable modifications to this methodology, we can achieve remarkable results for motion transitions - a topic which will be discussed in later sections.

\subsubsection{Partial Hybrid Zero Dynamics}

As discussed in Chapter 4, the goal of the control law $u_{\alpha}(4.5)$ is to drive the human-inspired output $y_{\alpha}(\theta, \dot{\theta}) \rightarrow 0$ exponentially at a rate of $\varepsilon$. Therefore, for the continuous dynamics, the controller renders the full zero dynamics surface:

$$
\mathbf{F} \mathbf{Z}_{\alpha}=\left\{(\theta, \dot{\theta}) \in T Q_{R}: y_{\alpha}(\theta, \dot{\theta})=\mathbf{0}, L_{f_{R}} y_{2, \alpha}(\theta)=\mathbf{0}\right\}
$$

While this surface is invariant for continuous dynamics, however, this is not the case for discrete dynamics. In particular, the discrete impacts in the system cause the state to be "thrown" off of the zero dynamics surface. Therefore, a hybrid system has full hybrid zero dynamics (FHZD) if the zero dynamics are invariant through impact: $\Delta^{R}\left(S^{R} \cap \mathbf{F} \mathbf{Z}_{\alpha}\right) \subset \mathbf{F Z}_{\alpha}[2]$.

While the realization of FHZD renders a lot of favorable properties for the system, it is quite difficult in the case of bipedal robotic walking since it would force the hybrid system to evolve on a 1-dimensional manifold. Therefore, we seek to enforce zero dynamics only for the relative degree 2 outputs. We refer to this as the partial zero dynamics surface, given by:

$$
\mathbf{P Z}_{\alpha}=\left\{(\theta, \dot{\theta}) \in T Q_{R}: y_{2, \alpha}(\theta)=\mathbf{0}, \quad L_{f_{R} y_{2, \alpha}}(\theta)=\mathbf{0}\right\}
$$


Particularly, we can define the partial hybrid zero dynamics (PHZD) as: $\Delta^{R}\left(S^{R} \cap\right.$ $\left.\mathbf{F Z}_{\alpha}\right) \subset \mathbf{P Z}_{\alpha}$. The consideration of this modification is that PHZD allows some "freedom" in the movement of the system to account for differences between the robot model and human. Moreover, since the only output that is not included in the partial zero dynamics surface is the output that forces the forward hip velocity to be constant, enforcing PHZD simply means that we allow the velocity of the hip to compensate for the shocks in the system due to impact. After constraining the system on the PHZD surface invariantly through impact, we will achieve a system evolving with a 2-dimensional zero dynamics manifold which is determined by the parameters $\alpha$ only. Note that the PHZD surface is consistent for all four mobile motion primitives; thus the conclusion obtained in [3] for walking on flat ground also applies for the remaining two motion primitives.

\subsubsection{Human-Inspired Optimization}

With the PHZD constraints in mind, the goal to develop the controller becomes to find parameters $\alpha_{v}^{*}$ that solve the following constrained optimization problem:

$$
\begin{aligned}
\alpha_{v}^{*}=\underset{\alpha_{v} \in \mathbb{R}^{22}}{\operatorname{argmin}} \operatorname{Cost}_{\mathrm{HD}}\left(\alpha_{v}\right) \\
\text { s.t } \quad \Delta^{R}\left(S_{h_{v}}^{R} \cap \mathbf{F} \mathbf{Z}_{\alpha_{v}}\right) \subset \mathbf{P} \mathbf{Z}_{\alpha_{v}}
\end{aligned}
$$

with Cost $_{\mathrm{HD}}$ being the same as that in (2.6) and $v \in\{f g, u s, d s, r n\}$. This is simply the optimization problem in (2.6) that is used to determine the parameters of the ECHF to give the best fit of the human walking functions to the human output data of three motion primitives, but subject to constraints that ensure PHZD. Note that $S_{h_{v}}^{R}$ are different for different motion primitives. This method can be applied to all three mobile motion primitives; the only difference is the height above the walking 
surface $h_{v}$, i.e., the guard.

In order to solve the (6.3) explicitly, we restate the PHZD constraints with applying the inverse kinematics methodology discussed in [2] in such a way that it can be practically solved. Specifically, because of the formula of the outputs we choose (all outputs are linear) and the way we parameterize the time, a point $(\vartheta(\alpha), \dot{\vartheta}(\alpha)) \in \mathbf{F Z}_{\alpha_{v}} \cap S_{h_{v}}^{R}$ on the intersection of the full zero dynamics surface and the switching surface can be explicitly computed in terms of the parameters $\alpha_{v}$ (detail derivation can be seen in [2]). Therefore, with the notation of partial zero dynamics surface and the point $(\vartheta(\alpha), \dot{\vartheta}(\alpha))$, we formally redefine the optimization problem with the PHZD constraints as the following human-inspired optimization problem theorem:

Theorem 1. The parameters $\alpha_{v}^{*}$ solving the constrained optimization problem:

$$
\begin{aligned}
\alpha_{v}^{*}=\underset{\alpha_{v} \in \mathbb{R}^{21}}{\operatorname{argmin}} & \operatorname{Cost}_{\mathrm{HD}}\left(\alpha_{v}\right) \\
\text { s.t } \quad & y_{2, \alpha}(\vartheta(\alpha))=\mathbf{0} \\
& d y_{2, \alpha}\left(\Delta_{\theta} \vartheta(\alpha)\right) \Delta_{\dot{\theta}}(\vartheta(\alpha)) \dot{\vartheta}(\alpha)=\mathbf{0} \\
& d h^{R}(\vartheta(\alpha)) \dot{\vartheta}(\alpha)<0
\end{aligned}
$$

yield hybrid zero dynamics: $\Delta^{R}\left(S_{h_{v}}^{R} \cap \mathbf{F Z}_{\alpha_{v}^{*}}\right) \subset \mathbf{P Z}_{\alpha_{v}^{*}}$. Furthermore, if

$$
\tau\left(\vartheta\left(\alpha^{*}\right)\right)=\frac{\delta p_{\text {hip }}\left(\vartheta\left(\alpha^{*}\right)\right)-\delta p_{\text {hip }}\left(\Delta_{q} \vartheta\left(\alpha^{*}\right)\right)}{v_{h i p, v}}>0
$$

then there exists a constant $\bar{\varepsilon}>0$ such that for all $\varepsilon>\bar{\varepsilon}$ the hybrid system $\mathscr{H}_{\left(h_{v}, \alpha_{v}^{*}\right)}^{R, m}$ has an exponentially stable periodic orbit. Moreover, the fixed point of this periodic 
orbit, $\left(\theta_{\varepsilon, v}^{*}, \dot{\theta}_{\varepsilon, v}^{*}\right)$, satisfies the property that:

$$
\lim _{\varepsilon \rightarrow \infty}\left(\theta_{\varepsilon, v}^{*}, \dot{\theta}_{\varepsilon, v}^{*}\right)=\left(\vartheta\left(\alpha^{*}\right), \dot{\vartheta}\left(\alpha^{*}\right)\right) .
$$

This Theorem follows from a combination of the Theorem 1 in [2] and Theorem 2 in [3], but extended to the case of different motion primitives. The proof of this theorem is also similar to the proof in those two papers; since this proof would require the introduction of numerous constructions that are not necessary to the rest of the results given in this thesis, we will not state it in explicit detail. The only difference is that for different motion primitives, we have different guards $h_{v}(q)$ - which renders the proof, for the most part, unchanged. The proof of the first part of Theorem 1 is the same as in [2] since PHZD is invariant corresponding to the height guard. For the proof of the second part, it is similar to the proof of Theorem 2 in [3] but with a constant shift in the Poincaré map for different motion primitives (different guards); however, these shifts do not affect the proof of the existence of the fixed point $\left(\vartheta\left(\alpha^{*}\right), \dot{\vartheta}\left(\alpha^{*}\right)\right)$. Note that, the Theorem 1 doesn't guarantee the convergence to the fixed point, but insures the existence of the fixed point if the optimization problem subject to the PHZD constraints can be solved. It's these special constraints which guarantee stable walking that are the highlights of this methodology. As we know that these constraints are highly nonlinear, it's difficult (if not impossible) to locate or to identify the global minimum. However, we argue that with sufficient small feasibility, we can conclude that a good solution which guarantees stable walking with PHZD, will be found. Feasibility, one of the outputs of MATLAB built in function fmincon, denotes the maximum constraint violation.

Now we can restate the main contribution of this thesis - starting from the human data, through the inverse kinematics and the parameters obtained from op- 


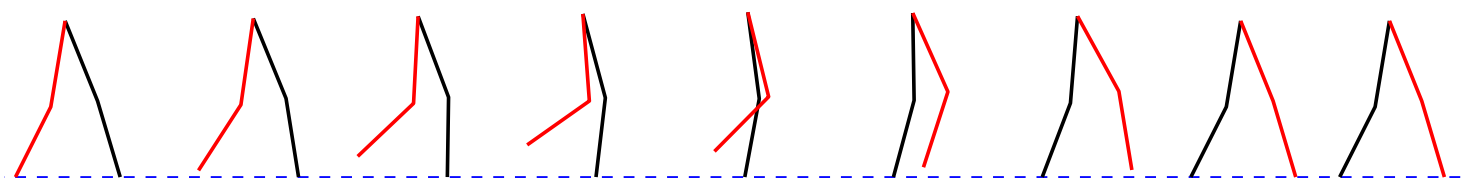

(a) Walking on flat ground

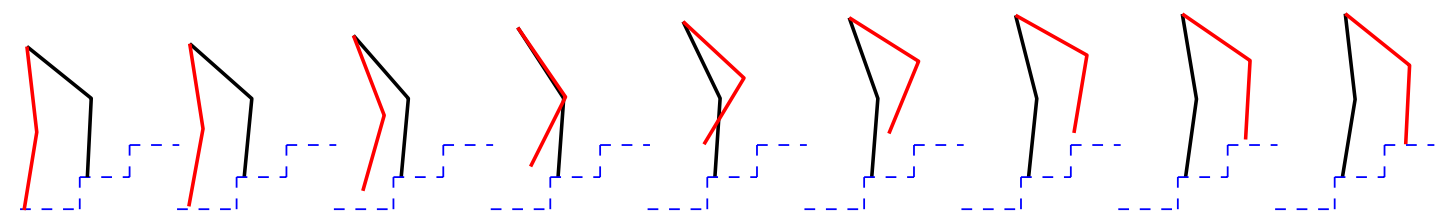

(b) Walking upstairs

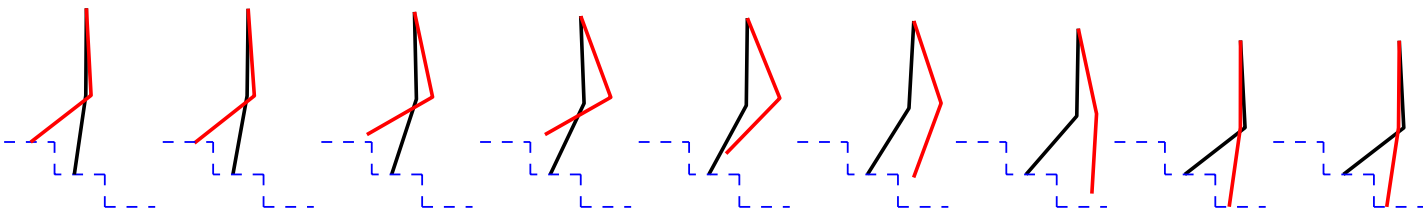

(c) Walking downstairs
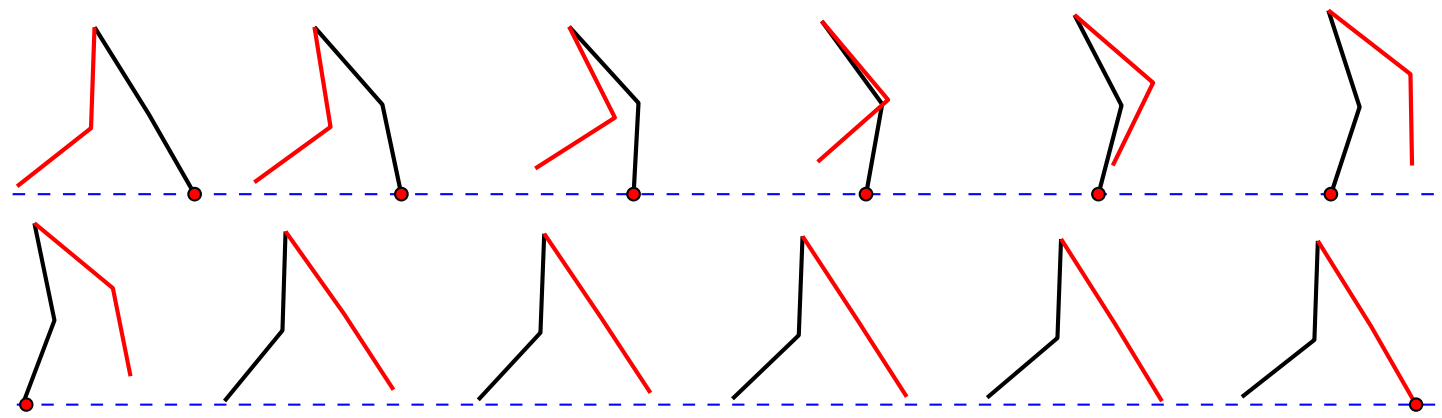

(d) Running

Figure 6.2: Snapshots from robotic locomotion simulations exhibiting the four motion primitives [38].

timization problem (6.4), we can determine the fixed points $\left(\vartheta\left(\alpha^{*}\right), \dot{\vartheta}\left(\alpha^{*}\right)\right)$, where $v \in\{f g, u s, d s, r n\}$, to the stable hybrid periodic orbits of all four mobile motion primitives. Since the cost function of the optimization problem only depends on the human walking data, we reinforce the fact that we can automatically generate a controller, which yields a stable walking gait and the fixed point of its stable hybrid 


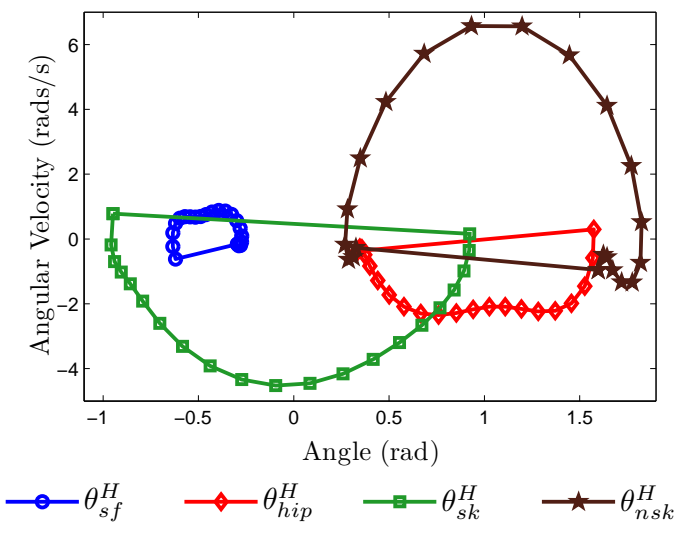

(a) Periodic orbit for $\mathscr{M}_{a s}$

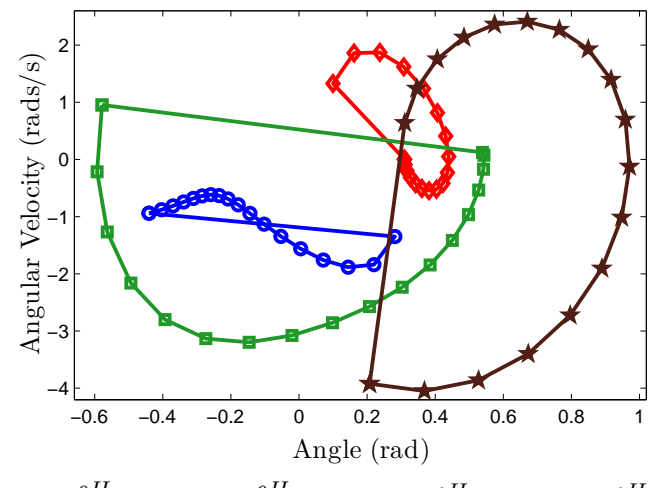

$\multimap \theta_{s f}^{H} \multimap \theta_{h i p}^{H} \longrightarrow \theta_{s k}^{H} \multimap \theta_{n s k}^{H}$

(c) Periodic orbit for $\mathscr{M}_{f g}$
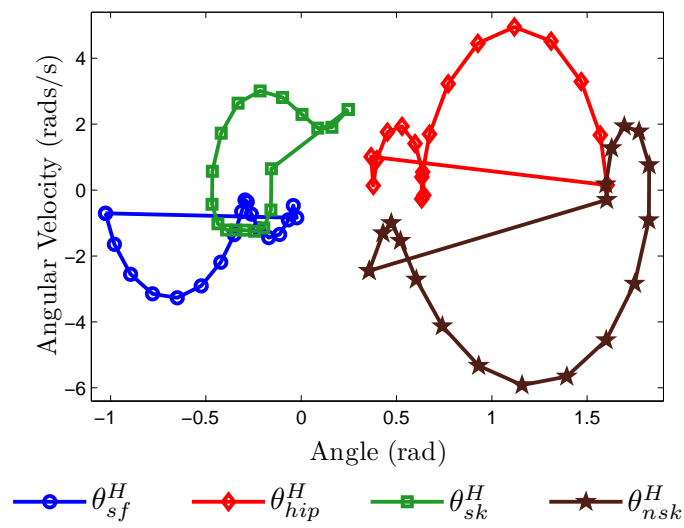

(b) Periodic orbit for $\mathscr{M}_{d s}$
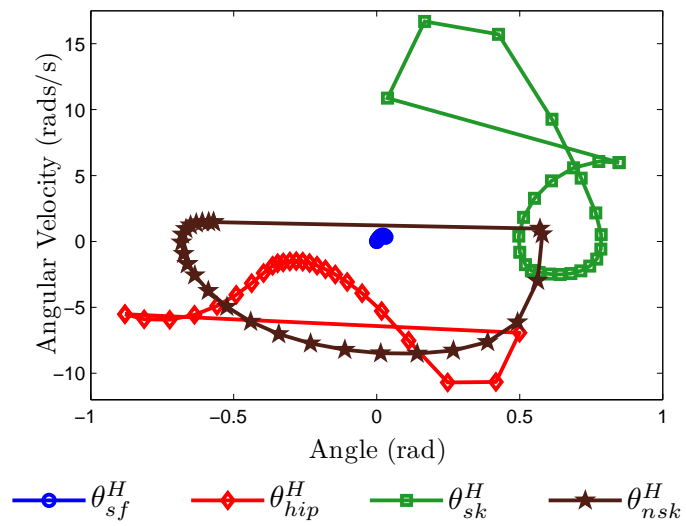

(d) Periodic orbit for $\mathscr{M}_{r n}$

Figure 6.3: Phase portraits for the four motion primitives.

periodic orbit, by only using the human walking data - for all four mobile motion primitives.

Remark. Theorem 1 only applies to the fully actuated motion primitives including level walking, stair ascending and stair descending. The running motion primitive consists of an underactuated flight phase, therefore, requiring a different approach. The theorem of running motion primitive can be referred to [39]. The stability of the running gait is numerically verified using the Poincaré map. 


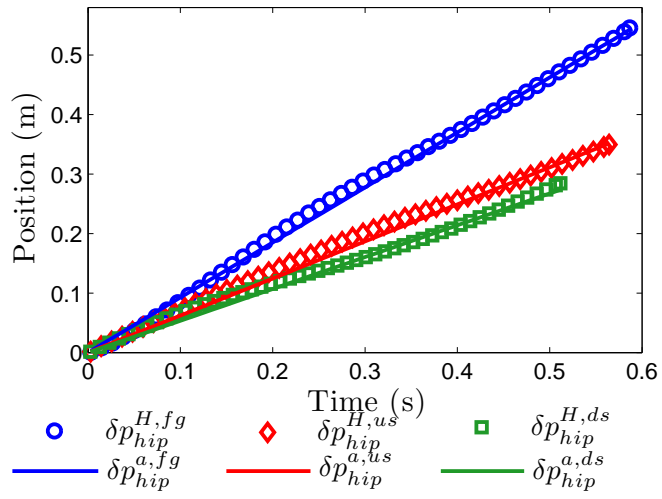

(a) Optimized hip position
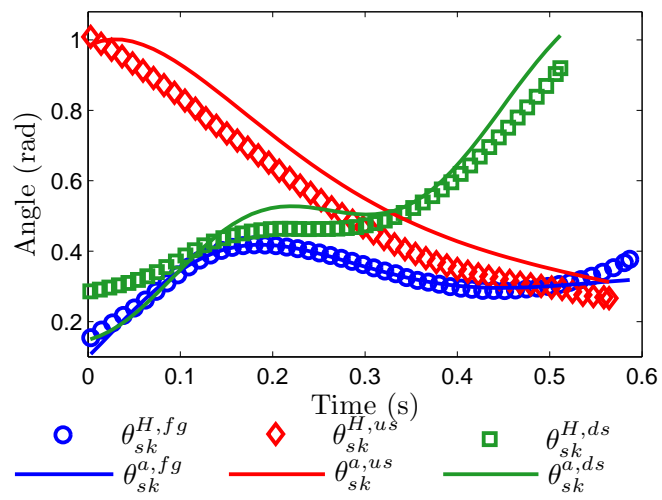

(c) Optimized stance knee angle

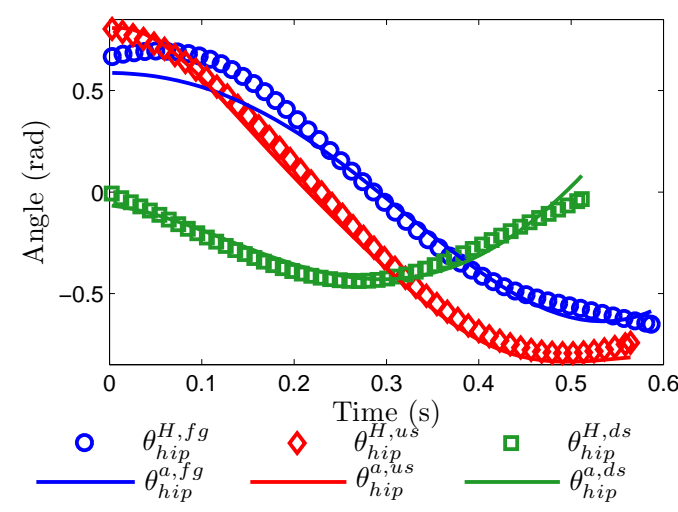

(b) Optimized nonstance hip angle

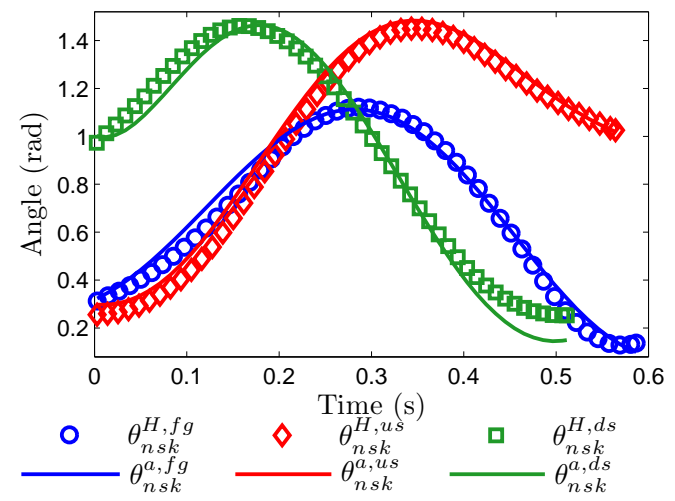

(d) Optimized nonstance knee angle

Figure 6.4: Optimized extended canonical human functions with parameters obtained by solving the optimization problem (2.6) and the corresponding mean human data of three one-phase motion primitives [38].

\subsubsection{Simulation Results}

Using the results of Theorem 1, which yields both the parameters for the humaninspired controller $\alpha_{v}^{*}$, along with a fixed point to the Poincaré map, we obtain stable walking for each motion primitive. Note that, the feasibility of each case is less than $1 e^{-10}$. The resulting locomotion gaits from simulation are given in Fig. 6.2; these figures show the evolution of the robot during the single support phase of the gait, each of which qualitatively resembles the corresponding human gait quite well. The phase portraits for each motion primitive simulation are shown in Fig. 6.3. The 


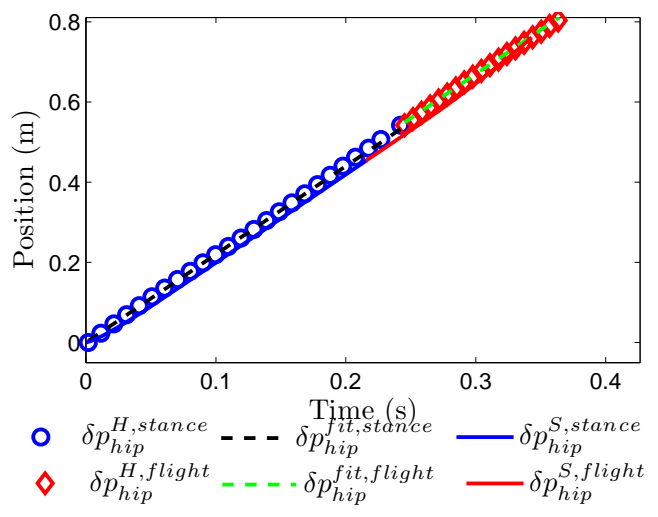

(a) Optimized hip position

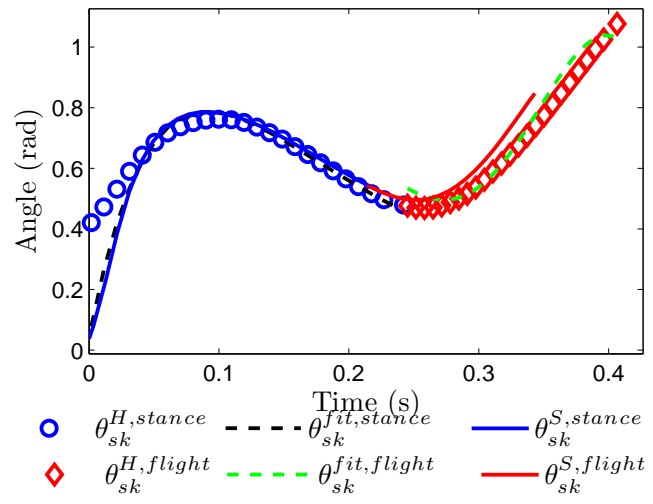

(c) Optimized stance knee angle

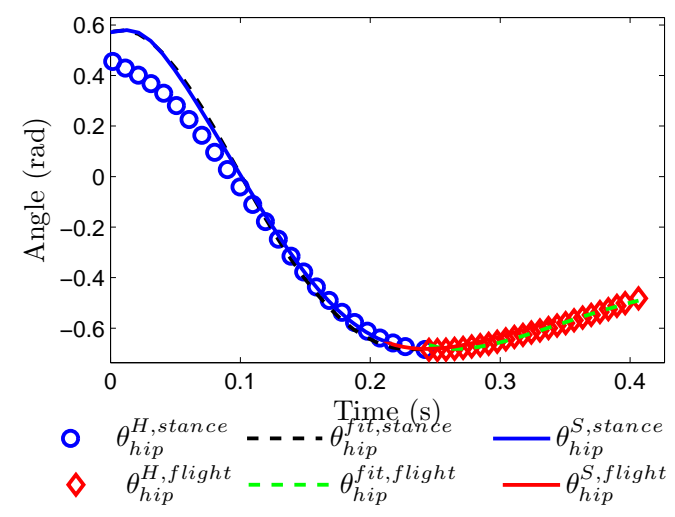

(b) Optimized nonstance hip angle

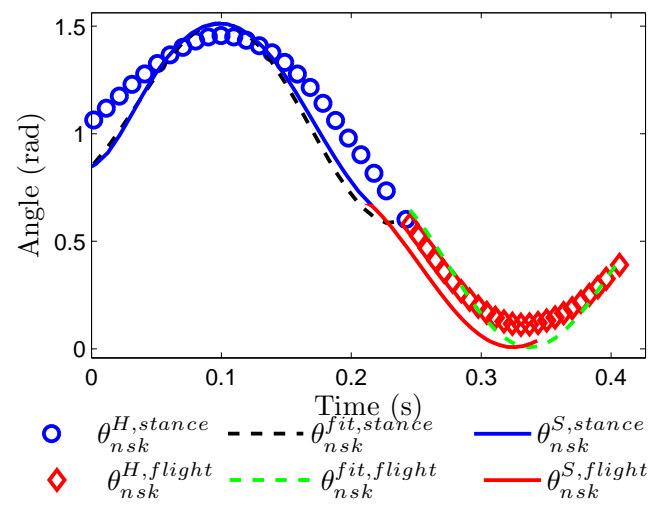

(d) Optimized nonstance knee angle

Figure 6.5: Optimized canonical human functions with parameters obtained by solving the optimization problem (2.6) and the corresponding mean human data of the two-phase running motion primitive [39].

stability of each phase portrait is numerically verified via numerical approximation of the eigenvalues of the Poincaré map. All maximum eigenvalues are less than one as shown in Fig. 6.8, which implies the corresponding motion primitives are stable. Note that, we also achieved bipedal robotic running (with speed of $2.5 \mathrm{~m} / \mathrm{s}$ ) using the human-inspired optimization with ECHF.

Along with stable walking, the simulation results show that the bipedal model considered in this thesis has achieved human-like walking for each mobile motion primitive. Table. 7.3 contains the specific parameters $\alpha_{v}^{*}$ obtained through opti- 
mization mentioned above for the one-phase motion primitives. The correlations are all higher than 0.96 , which are close to the fitting correlations; and costs from optimization are low. The parameters $\alpha_{r n}^{*}$ for the two-phase running motion primitive are shown in Table. 7.4. Plots of the human walking functions with these parameters, as compared against the human data, can be seen in Fig. 6.4 for three one-phase motion primitives and Fig. 6.5 for the two-phase running motion primitive. From the figure and the table, one can conclude that the bipedal robot considered in this thesis, with human inspired locomotion controller, has achieved qualitatively human-like walking. It is also important to note that the velocities after impact for all the one-phase motion primitives are below $7 \mathrm{rad} / \mathrm{s}$, which is realistic in a physical context.

\subsection{Motion Transitions}

This section discusses the development and simulation of motion transitions, which are explicitly built upon the motion primitives obtained in the previous section. Note that, because of the lacking of transition data between level walking and running, this section will focus on the frame work of obtaining transitions between the one-phase motion primitives. The transitions between level walking and running will be achieved with a different approach, the derivation of which is omitted in this work in order to keep the simplicity of the thesis structure.

\subsubsection{Motion Transition Collection}

We are interested in developing motion primitives based upon the meta-hybrid system graph $\Gamma^{R}$, which gives the allowable transitions between different locomotion behaviors. Based upon the definition of a meta-hybrid system (Definition 1), the motion transitions must satisfy very specific conditions with regard to the motion primitives. Therefore, specific motion transition hybrid systems we are interested in 
must have the form:

- Standing still to walking on flat ground: $\mathscr{H}_{\left(h_{f g}, \alpha_{(s s, f g)}\right)}^{R}$, where $h_{f g}(q)=z_{n s f}(q)$,

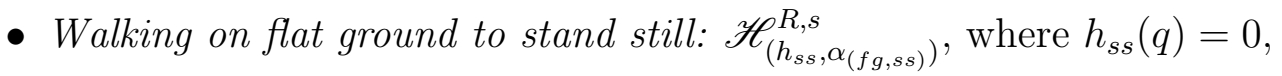

- Walking on flat ground to up stairs: $\mathscr{H}_{\left(h_{u s}, \alpha_{(f g, u s)}\right)}^{R, m}$, where $h_{u s}(q)=z_{n s f}(q)-$ $z_{\text {stair }}$

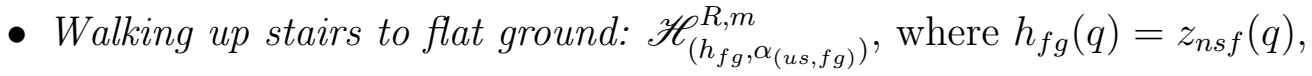

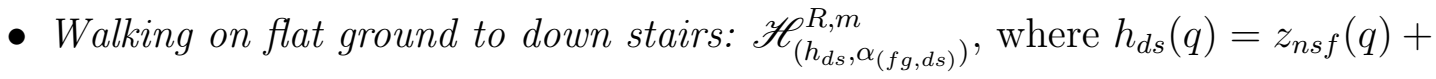
$z_{\text {stair }}$

- Walking down stairs to flat ground: $\mathscr{H}_{\left(h_{f g}, \alpha_{(d s, f g)}\right)}^{R,}$ where $h_{f g}(q)=z_{n s f}(q)$.

Since we have two types of motion primitives, the methodology to determine the control parameters $\alpha_{e}^{*}, e \in E$ corresponding to different transitions will be different. Therefore, we separate the 6 motion transitions into two groups: the first group contains two motion transitions related to the stand still motion primitive, which are $\mathscr{H}_{\left(h_{f g}, \alpha_{(s s, f g)}\right)}^{R, s}$ and $\mathscr{H}_{\left(h_{s s}, \alpha_{(f g, s s)}\right.}^{R, s}$; the remaining four motion transitions belong to the second group. We name the first group as "stationary related motion transitions" and the second group as "mobile related motion transitions". In the following section, we discuss the optimization problems through which the motion transitions are obtained.

\subsubsection{Controller Development}

Motion transitions connect two different motion primitives. Therefore, we have to take both source and target motion primitives into consideration while we are constructing optimizations for motion transitions.

Stationary Related Motion Transitions. To determine the parameters $\alpha_{e}^{*}$, $e \in E$, of the stationary related motion transitions we use the fixed points cor- 


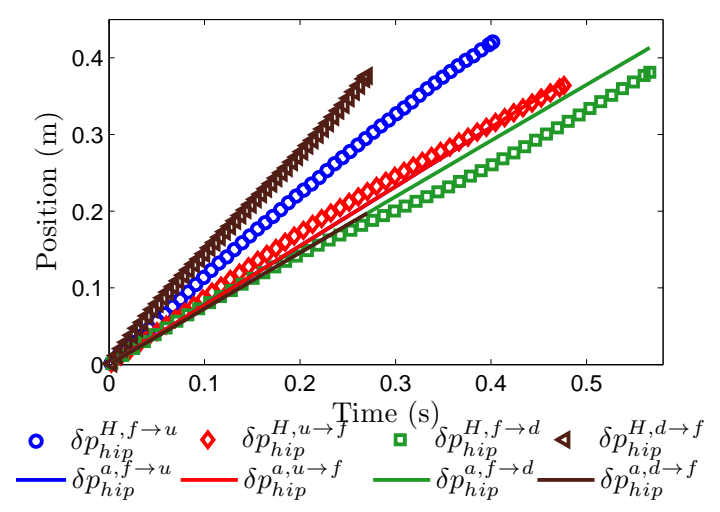

(a) Optimized hip position

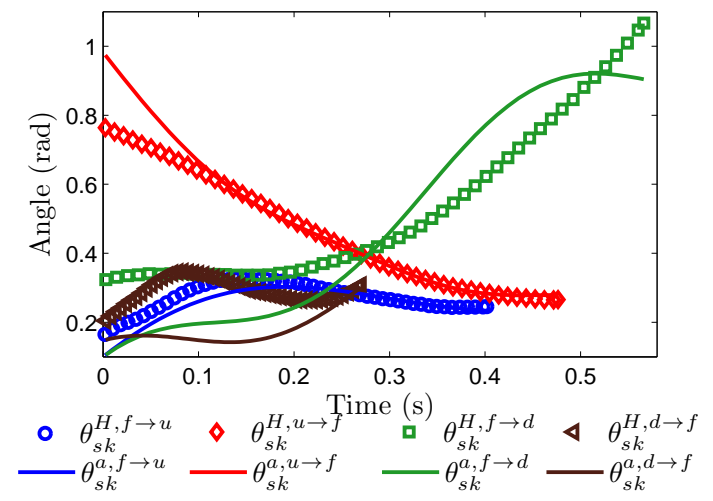

(c) Optimized stance knee angle

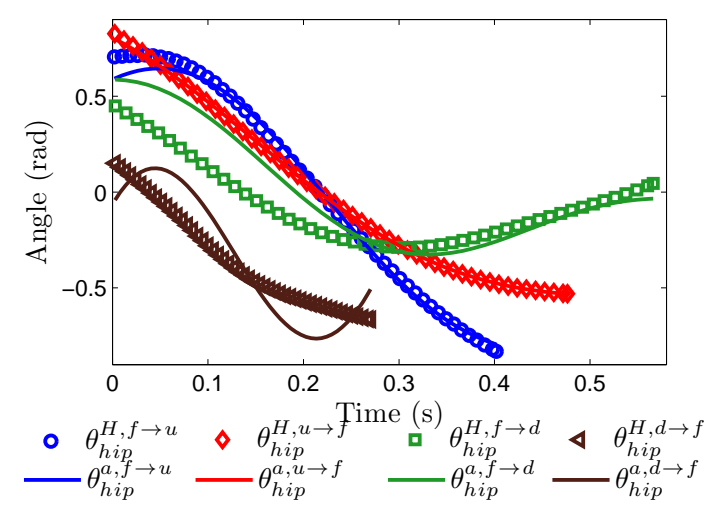

(b) Optimized nonstance hip angle

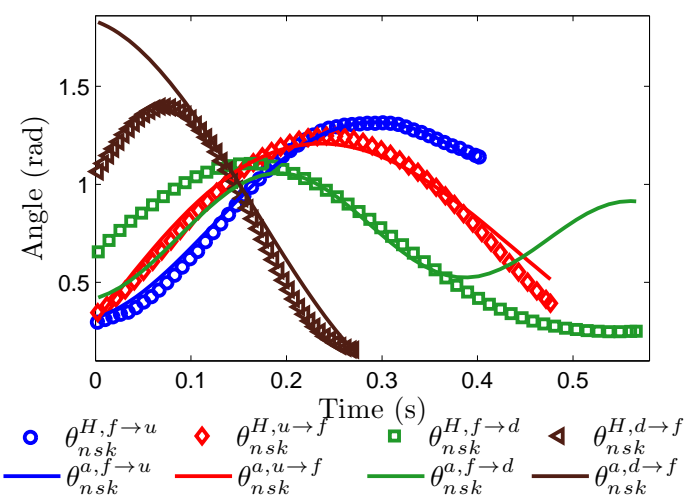

(d) Optimized nonstance knee angle

Figure 6.6: Optimized extended canonical human functions with parameters obtained by solving the optimization problem (2.6) and the corresponding mean human data of four motion transitions [38].

responding to motion primitives of standing still on flat ground and flat ground walking. In particular, $\left(\vartheta\left(\alpha^{*}\right), \dot{\vartheta}\left(\alpha^{*}\right)\right) \in S_{h_{v}}^{R}, v \in V^{R}$ (here, $\left.v \in\{s s, f g\}\right)$, are the fixed point of each motion primitive, which are computed via the Theorem 1 in closed form. The second main contribution of this thesis can now be stated: since we can explicitly compute the fixed points of periodic orbits of all three mobile motion primitives with Theorem 1, we propose that utilizing the fixed points obtained above in an optimization problem can also render the parameters of controllers for motion transitions. At a high level, the goal of the motion transition optimization is to gen- 
erate desired output functions, which effect smooth connections, or "transitions", between the corresponding source and the target motion primitives. Formally, these objectives can be stated in an optimization problem:

$$
\begin{gathered}
\alpha_{e}^{*}=\underset{\alpha \in \mathbb{R}^{22}}{\operatorname{argmin}}\left(\left\|\left(\vartheta_{e}\left(\alpha_{e}\right), \dot{\vartheta}_{e}\left(\alpha_{e}\right)\right)-\left(\vartheta_{\operatorname{tar}(e)}\left(\alpha_{\operatorname{tar}(e)}^{*}\right), \dot{\vartheta}_{\operatorname{tar}(e)}\left(\alpha_{\operatorname{tar}(e)}^{*}\right)\right)\right\|\right) \\
\text { s.t } \quad y_{2, \alpha_{e}}^{d}(0)-y_{2, \alpha_{s o r(e)}^{*}}^{d}(0)=0 \\
\dot{y}_{2, \alpha_{e}}^{d}(0)-\dot{y}_{2, \alpha_{s o r(e)}^{*}}^{d}(0)=0
\end{gathered}
$$

where $y_{2, \alpha_{e}}^{d}(t)$ are the desired relative degree two outputs of the robot with parameters $\alpha_{e} ; \alpha_{\operatorname{tar}(e)}^{*}$ and $\alpha_{\mathrm{sor}(e)}^{*}$ are the optimized parameters of the target and source motion primitives, $\mathscr{M}_{\mathrm{tar}(e)}^{R}$ and $\mathscr{M}_{\mathrm{sor}(e)}^{R}$, respectively. They are obtained explicitly by solving the optimization problem (6.4) and $\left(\vartheta_{\operatorname{tar}(e)}\left(\alpha_{\operatorname{tar}(e)}^{*}\right), \dot{\vartheta}_{\operatorname{tar}(e)}\left(\alpha_{\operatorname{tar}(e)}^{*}\right)\right)$ is the fixed point of target motion primitive. Note that, here $\operatorname{tar}(e), \operatorname{sor}(e) \in\{s s, f g\} .\left(\vartheta_{e}\left(\alpha_{e}\right), \dot{\vartheta}_{e}\left(\alpha_{e}\right)\right)$ is the pre-impact point of the end of the transition, which is the point at the intersection of the full zero dynamics surface of the transition and the guard of the target motion primitive (thus, this point can be computed via the methodology of inverse kinematics). Through the cost function, we enforce the end point of the transition to be as close to the fixed point of the target motion primitive as possible, while constraining the transition to start with fixed point of the source motion primitive. Some augmentation constraints, such as angle limits, have been added to insure the transition behavior is as human-like as possible. Solving this optimization renders the parameters $\alpha_{e}^{*}$ for the two stationary motion transitions.

Mobile Related Motion Transitions. To generate the parameters $\alpha_{e}^{*}, e \in E$, of the mobile related motion transitions, we turn to a different approach. As we have the human data for these four motion transitions, we use the same cost function as in 
(2.6) with the constraints corresponding to the target and source motion primitives. The problem can be stated as:

$$
\begin{aligned}
& \alpha_{e}^{*}=\underset{\alpha \in \mathbb{R}^{22}}{\operatorname{argmin}} \operatorname{Cost}_{\mathrm{HD}}\left(\alpha_{e}\right) \\
& \text { s.t } \quad y_{2, \alpha_{e}}^{d}(0)-y_{2, \alpha_{\text {sor }(e)}^{*}}^{d}(0)=0 \\
& \dot{y}_{2, \alpha_{e}}^{d}(0)-\dot{y}_{2, \alpha_{\text {sor }(e)}^{d}}^{d}(0)=0 \\
& y_{2, \alpha_{e}}^{d}\left(\tau_{\alpha_{e}, \alpha_{\operatorname{tar}(e)}^{*}, \alpha_{\text {sor }(e)}^{*}}\right)-y_{2, \alpha_{\operatorname{tar}(e)}^{*}}^{d}\left(\tau_{\alpha_{e}, \alpha_{\operatorname{tar}(e)}^{*}, \alpha_{\text {sor }(e)}^{*}}\right)=0 \\
& \dot{y}_{2, \alpha_{e}}^{d}\left(\tau_{\alpha_{e}, \alpha_{\operatorname{tar}(e)}^{*}, \alpha_{\text {sor }(e)}^{*}}\right)-\dot{y}_{2, \alpha_{\operatorname{tar}(e)}^{*}}^{d}\left(\tau_{\alpha_{e}, \alpha_{\operatorname{tar}(e)}^{*}, \alpha_{\text {sor }(e)}^{*}}\right)=0 \\
& \tau_{\alpha_{e}, \alpha_{\text {tar }(e)}^{*}, \alpha_{\text {sor }(e)}^{*}}=\frac{\delta p_{\text {hip }}^{R}\left(\vartheta_{\operatorname{tar}(e)}\left(\alpha_{\operatorname{tar}(e)}^{*}\right)\right)-\delta p_{\text {hip }}^{R}\left(\Delta_{q} \vartheta_{\text {sor }(e)}\left(\alpha_{\text {sor }(e)}^{*}\right)\right)}{v_{\text {hip }, e}}
\end{aligned}
$$

where the elements are the same as those in (6.6) except that $\operatorname{tar}(e), \operatorname{sor}(e) \in$ $\{f g, u s, d s\} ; \operatorname{Cost}_{\mathrm{HD}}\left(\alpha_{e}\right)$ is the cost given in (2.6) and $e \in E^{R}$. Note that $\tau_{\alpha_{e}, \alpha_{\text {tar }(e)}^{*}, \alpha_{s o r(e)}^{*}}$ is an approximation of the transition time interval, which is computed with the assumption that the hip velocity of the transition behavior is constant. Examination of human data for the hip position, given in Fig. 2.4a, reveals that this assumption is reasonable. $\delta p_{\text {hip }}^{R}\left(\vartheta_{\operatorname{tar}(e)}\left(\alpha_{\operatorname{tar}(e)}^{*}\right)\right)$ and $\delta p_{\text {hip }}^{R}\left(\Delta_{q} \vartheta_{\text {sor }(e)}\left(\alpha_{\text {sor }(e)}^{*}\right)\right)$ are the pre-impact hip position of target motion primitive and the post-impact hip position of source motion primitive, respectively. And $v_{h i p, e}$ is the actual hip velocity of the specified motion transition. The optimization here constrains both start and end points of transitions to be the fixed points of source and target modes, respectively. From a geometric view, we can interpret this process as constructing a topology transformation going smoothly from one convex invariant set to another convex invariant set. By solving this optimization problem, we obtain parameters $\alpha_{e}^{*}$ for all four motion transitions.

In this framework, the motion transitions play an important role in the motion planning of the robot, i.e., switching between different motion primitives. Rather 
than switching directly between different motion primitives (ZMP is used in [21], central pattern generator is used [16]), we utilize the motion transition as a "buffer" to connect the fixed point of each motion primitive to insure the stability of system while switching. One main advantage of the introduction of motion transitions is that the system can deal with big variation of the terrain with the guarantee of good stability. Another advantage is that the motion transition generates smooth human-like switching process without using high gain and won't generate torque spikes.

This approach is different from funneling work [6], in which the controllable state space is covered with the regions of attraction from a lot of locally stabilizing controllers to insure robustness of the transitions. Therefore, the motion planning can be considered as switching between different funnels which, for example, can be computed numerically using the method in [31]. Compared to all the pioneering funneling works $[6,12,18]$, we focus more on constructing a directed graph consisting nodes (motion primitives) and edges (motion transitions) instead of building funnels. Then the motion can be planned in the directed graph through switching among the designed nodes via the corresponding edges. Since the motion transitions are optimized in such a way that the fixed point of motion primitives which are asymptotically stable, can be connected directly and smoothly, the stability of the motion planning can be guaranteed naturally. Particularly, the numerical method of Poincaré map is used to prove the stability of the transition behaviors, which will be discussed later.

Note that, as in the case of the optimization for PHZD, we only constrain the relative degree two outputs. Thus, some "freedom" is given to the robot to compensate for the differences between the robot and the physical human body. Solving these two optimization problems yields parameters $\alpha_{e}^{*}, e \in E^{R}$; therefore, yields the motion transition hybrid system: $\mathscr{T}_{e}=\mathscr{H}_{\left(h_{\operatorname{tar}(e)}^{R, i}, \alpha_{e}^{*}\right)}$ with $e \in E^{R}$ and $i \in\{s, m\}$. 


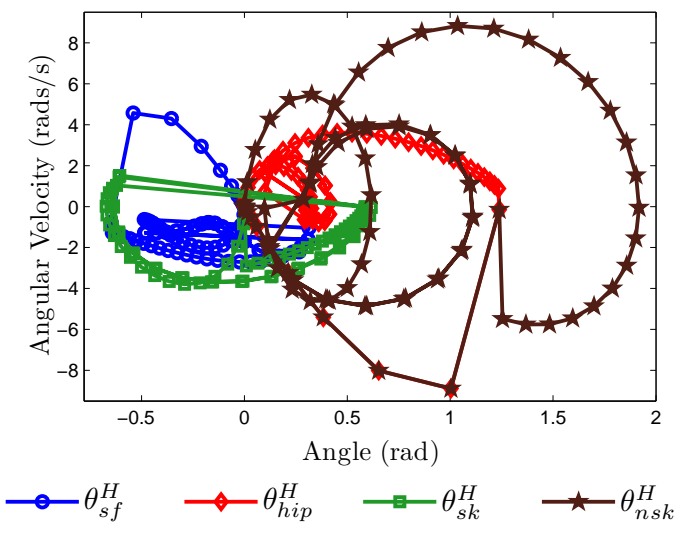

(a) Periodic orbit for $\mathscr{H} \mathscr{M}_{\{f g, s s\}}$

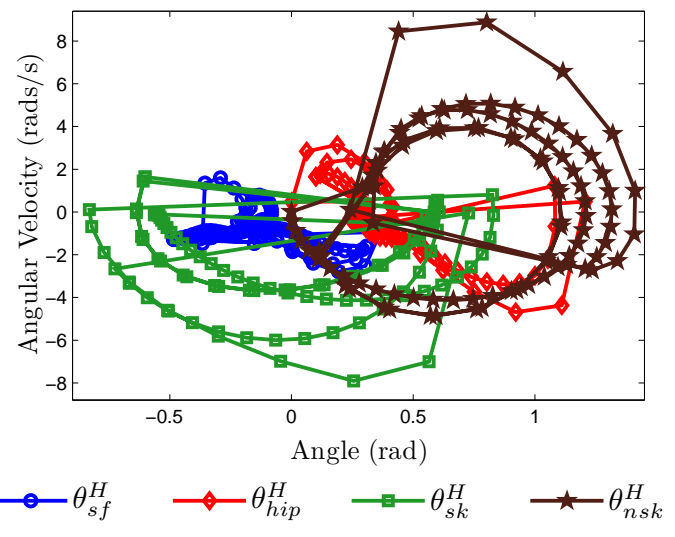

(b) Periodic orbit for $\mathscr{H} \mathscr{M}_{\{f g, u s\}}$

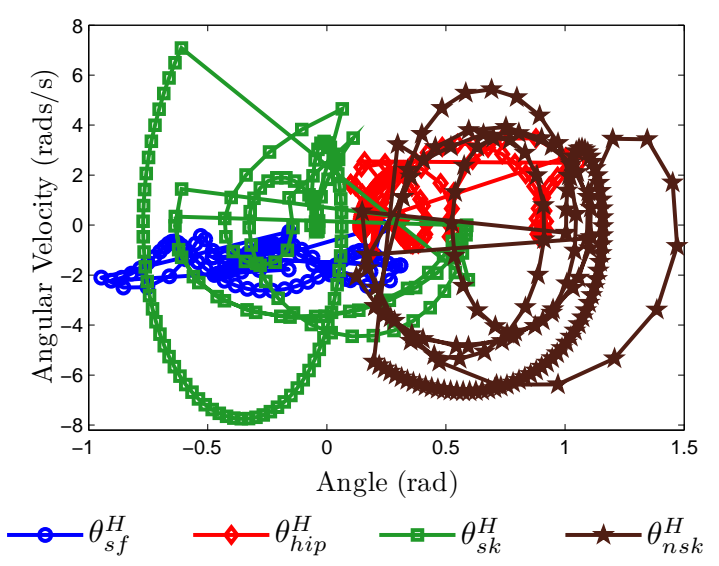

(c) Periodic orbit for $\mathscr{H} \mathscr{M}_{\{f g, d s\}}$

Figure 6.7: Phase portraits for the combination of motion primitives and motion transitions [38].

\subsubsection{Simulations Results}

For the mobile related motion transitions, the simulation results show that the robot considered in this thesis achieves human-like motion transitions. Table 7.3 contains the specific $\alpha_{e}^{*}$ obtained through the optimization for each mobile related motion transition. The correlations of transitions related to going up stairs are all higher than 0.98 , and the costs are on par with the costs of the motion primitives. 


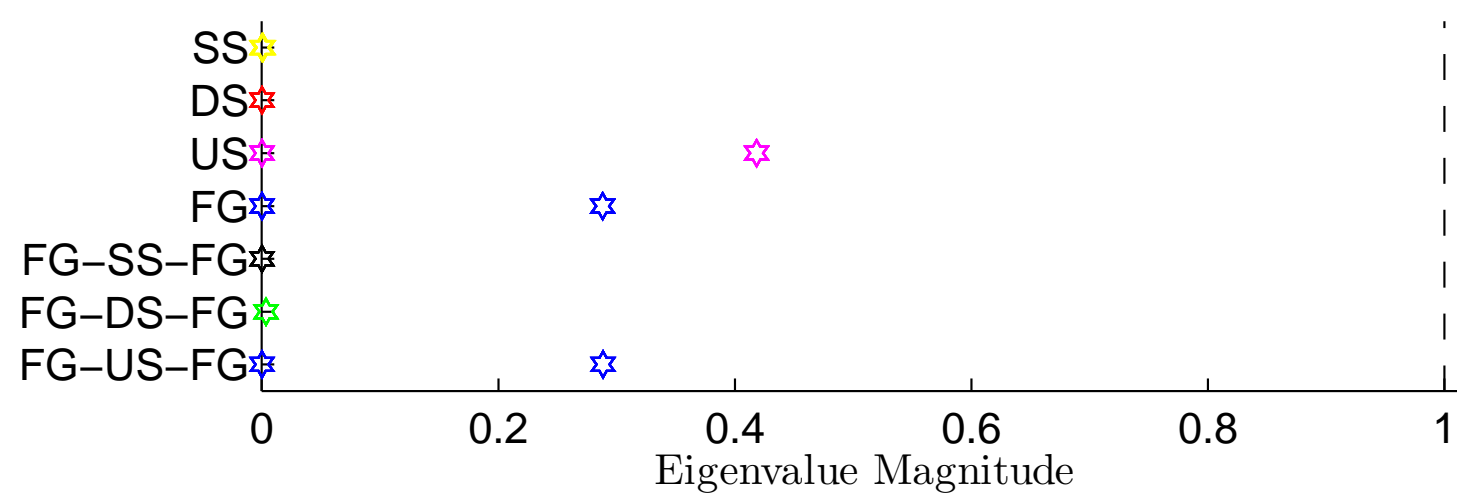

Figure 6.8: Eigenvalues of motion primitives [38].

For the transitions corresponding to going downstairs, the correlations are still high (higher than 0.93) except for one output. This could be related to the fact that the experimental human data of going downstairs contains a relatively high amount of noise. Plots of the human walking functions with these parameters, as compared against human data, can be seen in Fig. 6.6.

Three simulations were performed in which the one-phase motion primitives and motion transitions were combined. To construct a Poincaré map, and thus establish a notion of the stability of a meta-system, the biped must start and end in the same mode; therefore, we chose to simulate three locomotion cycles: walking on flat ground to stand still to walking on flat ground (FG-SS-FG), walking on flat ground to walking up stairs to walking on flat ground (FG-US-FG) and walking on flat ground to walking down stairs to walking on flat ground (FG-DS-FG). Numerical approximation yields eigenvalues for all three simulations; the maximum eigenvalue of each is below unity which implies that all three meta-systems are stable. The phase portraits of these three meta-systems can be seen in Fig.6.7, from which it can be seen that all velocities after impact for all three meta-systems are below 10 $\mathrm{rad} / \mathrm{s}$, which is reasonably feasible in practice. Finally, we simulated all four one- 


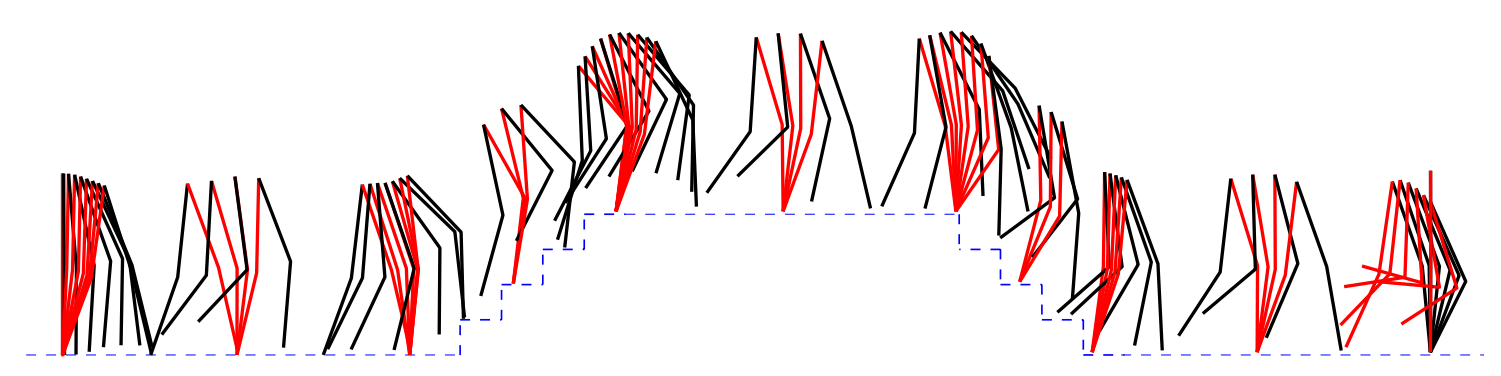

Figure 6.9: Snapshots from the simulated composition of multiple locomotion modes [38].

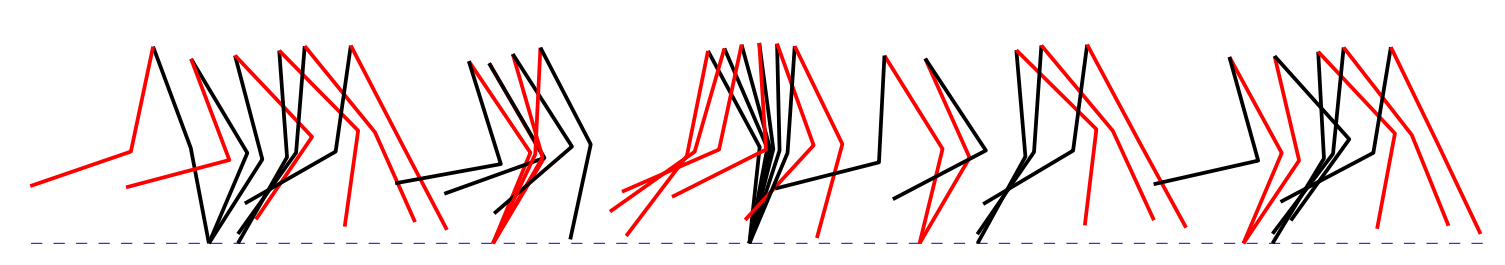

Figure 6.10: Snapshots from the simulated transitions between running and level walking.

phase motion primitives together with the six motion transitions; snapshots from the simulation can be seen in Fig. 6.9.

To achieve the transitions between flat ground walking and running, we take the approach of using transition matrix, which has been first proposed and realized in a physical bipedal robot in [19]. The snapshots of simulating running to walking and back to running is shown in Fig. 6.10. 


\section{SUMMARY}

In this thesis, the kinematic human locomotion data are examined on four modes of locomotion. It is shown that certain outputs of the human locomotion data can each be represented by the response of a linear spring-damper system (for flat ground walking and running) or the response of a linear spring-damper system with a sinusoidal excitation (for stair ascent and descent). An optimization problem is proposed to give virtual outputs for feedback linearization controller, which yield provably stable and periodic locomotion in simulation. A second optimization is introduced to generate controllers which yield smooth motion transitions between motion primitives. Simulations are given which display bipedal robots walking in a varying terrain. Future work will be devoted to realizing the results of this work on a physical bipedal robot. 
Table 7.1: Fitted parameter values for human functions.

\begin{tabular}{|c|c|c|c|c|c|c|c|c|}
\hline \multicolumn{9}{|c|}{$y_{1}^{d}=a_{1} t, \quad y_{2}^{d}=y_{H}(t)$ given in $(2.4)$} \\
\hline f. & $a_{1}$ & $a_{2}$ & $a_{3}$ & $a_{4}$ & $a_{5}$ & $a_{6}$ & $a_{7}$ & Corr. \\
\hline$\delta p_{\text {hip }}^{f g}$ & 0.934 & 0 & 0 & 0 & 0 & 0 & 0 & 0.9991 \\
\hline$\delta p_{\text {hip }}^{u s}$ & 0.639 & 0 & 0 & 0 & 0 & 0 & 0 & 0.9954 \\
\hline$\delta p_{\text {hip }}^{d s}$ & 0.546 & 0 & 0 & 0 & 0 & 0 & 0 & 0.9976 \\
\hline$\theta_{h i p}^{f g}$ & 2.675 & 0.981 & 6.063 & 0.937 & 0 & 0 & -0.353 & 0.9991 \\
\hline$\theta_{\text {hip }}^{u s}$ & 2.093 & 1.192 & 6.039 & 0.286 & -0.038 & 12.572 & -0.353 & 1.0000 \\
\hline$\theta_{\text {hip }}^{d s}$ & 0.103 & 0.158 & 8.167 & -0.238 & -0.009 & 22.08 & -0.153 & 0.9999 \\
\hline$\theta_{s k}^{f g}$ & 3.322 & -0.174 & 13.66 & 0.040 & 0 & 0 & 0.333 & 0.9934 \\
\hline$\theta_{s k}^{u s}$ & 4.402 & 0.787 & 3.318 & 0.543 & -0.010 & 16.09 & 0.236 & 1.0000 \\
\hline$\theta_{s k}^{d s}$ & 0.548 & -10.34 & 0.535 & -11.35 & -0.065 & 17.33 & 10.70 & 0.9992 \\
\hline$\theta_{n s k}^{f g}$ & -0.861 & -0.344 & 10.57 & 0.046 & 0 & 0 & 0.681 & 0.9996 \\
\hline$\theta_{n s k}^{u s}$ & -2.059 & 0.276 & 0.074 & 1.072 & -0.409 & 10.90 & 0.407 & 0.9999 \\
\hline$\theta_{n s k}^{d s}$ & 2.667 & 1.264 & 3.460 & 2.197 & -0.180 & 13.90 & -0.172 & 0.9998 \\
\hline$\delta p_{\text {hip }}^{f \rightarrow u}$ & 1.085 & 0 & 0 & 0 & 0 & 0 & 0 & 0.9993 \\
\hline$\delta p_{\text {hip }}^{u \rightarrow f}$ & 0.799 & 0 & 0 & 0 & 0 & 0 & 0 & 0.9981 \\
\hline$\delta p_{\text {hip }}^{f \rightarrow d}$ & 0.668 & 0 & 0 & 0 & 0 & 0 & 0 & 0.9990 \\
\hline$\delta p_{h i p}^{d \rightarrow f}$ & 0.913 & 0 & 0 & 0 & 0 & 0 & 0 & 0.9996 \\
\hline$\theta_{h i p}^{f \rightarrow u}$ & -3.215 & 0.331 & 7.876 & -0.045 & 0.087 & 16.66 & 0.276 & 1.0000 \\
\hline$\theta_{h i p}^{u \rightarrow f}$ & 0.444 & 0.186 & 1.747 & -1.741 & 0.160 & 8.610 & 0.490 & 1.0000 \\
\hline$\theta_{h i p}^{f \rightarrow d}$ & -0.227 & -0.102 & 4.192 & -0.697 & 0.064 & 13.58 & 0.495 & 1.0000 \\
\hline$\theta_{\text {hip }}^{d \rightarrow f}$ & -2.480 & -0.947 & 1.930 & 0.041 & 0.044 & 16.87 & 1.050 & 0.9999 \\
\hline$\theta_{s k}^{f \rightarrow u}$ & 0.705 & -0.191 & 9.239 & 0.872 & 0.140 & 9.420 & 0.200 & 0.9940 \\
\hline$\theta_{s k}^{u \rightarrow f}$ & 3.820 & 0.514 & 4.154 & 0.185 & -0.009 & 17.24 & 0.261 & 1.0000 \\
\hline$\theta_{s k}^{f \rightarrow d}$ & -1.307 & -0.556 & 2.804 & 0.069 & -0.010 & 22.05 & 0.914 & 0.9997 \\
\hline$\theta_{s k}^{d \rightarrow f}$ & 1.057 & -0.703 & 5.593 & 1.094 & 0.611 & 6.759 & 0.278 & 0.9901 \\
\hline$\theta_{n s k}^{f \rightarrow u}$ & 0.217 & -0.473 & 9.417 & -0.021 & -0.128 & 15.81 & 0.908 & 0.9999 \\
\hline$\theta_{n s k}^{u \rightarrow f}$ & 1.691 & -0.335 & 7.973 & -0.265 & -0.019 & 8.197 & 0.677 & 0.9999 \\
\hline$\theta_{n s k}^{f \rightarrow d}$ & 0.919 & 0.112 & 8.101 & 0.574 & -0.034 & 16.88 & 0.567 & 1.0000 \\
\hline$\theta_{n s k}^{d \rightarrow f}$ & 1.301 & 0.372 & 10.82 & -0.024 & -0.106 & 11.14 & 0.777 & 0.9999 \\
\hline
\end{tabular}


Table 7.2: Fitted parameter values for human functions of running.

\begin{tabular}{|c|c|c|c|c|c|c|c|c|}
\hline \multicolumn{7}{|c|}{$y_{1}^{d}=v_{\text {hip }} t, y_{2}^{d}=y_{H}(t)$ given in $(2.4)$} \\
\hline$*$ & $*$ & $v_{\text {hip }}$ & $\alpha_{1}$ & $\alpha_{2}$ & $\alpha_{3}$ & $\alpha_{4}$ & $\alpha_{5}$ & Corr \\
\hline Mean & $\delta p_{\text {hip }}^{\text {stance }}$ & 2.327 & $*$ & $*$ & $*$ & $*$ & $*$ & 0.9999 \\
\hline$*$ & $\delta p_{\text {hip }}^{\text {flight }}$ & 2.440 & $*$ & $*$ & $*$ & $*$ & $*$ & 0.9998 \\
\hline$*$ & $\theta_{\text {hip }}^{\text {stance }}$ & $*$ & 1.319 & 0.627 & 15.29 & 0.023 & -0.110 & 1.0000 \\
\hline$*$ & $\theta_{\text {hip }}^{\text {flight }}$ & $*$ & -6.459 & 0.285 & 0.002 & 0.191 & -0.827 & 0.9998 \\
\hline$*$ & $\theta_{\text {sk }}^{\text {stance }}$ & $*$ & 7.502 & -0.374 & -0.000 & 0.061 & 0.916 & 0.9996 \\
\hline$*$ & $\theta_{\text {sk }}^{\text {flight }}$ & $*$ & 11.29 & -0.593 & 5.337 & -0.590 & 1.538 & 1.0000 \\
\hline$*$ & $\theta_{\text {nsk }}^{\text {stance }}$ & $*$ & -1.953 & -0.049 & 16.31 & 0.354 & 0.939 & 1.0000 \\
\hline$*$ & $\theta_{\text {nsk }}^{\text {flight }}$ & $*$ & 13.35 & 0.134 & 21.65 & -0.313 & 0.507 & 0.9999 \\
\hline
\end{tabular}


Table 7.3: Optimized parameter values and cost for human functions.

\begin{tabular}{|c|c|c|c|c|c|c|c|c|c|}
\hline \multicolumn{10}{|c|}{$y_{1}^{a}=a_{1} t, \quad y_{2}^{a}=y_{H}(t)$ given in $(2.4)$} \\
\hline f. & $a_{1}$ & $a_{2}$ & $a_{3}$ & $a_{4}$ & $a_{5}$ & $a_{6}$ & $a_{7}$ & Corr. & Cost \\
\hline$\delta p_{\text {hip }}^{f g}$ & 0.925 & 0 & 0 & 0 & 0 & 0 & 0 & 0.9991 & $*$ \\
\hline$\theta_{\text {hip }}^{f g}$ & -1.941 & 0.320 & 5.865 & -0.109 & 0 & 0 & 0.267 & 0.9958 & $*$ \\
\hline$\theta_{s k}^{f g}$ & 4.852 & -0.223 & 12.01 & 0.125 & 0 & 0 & 0.323 & 0.9787 & $*$ \\
\hline$\theta_{n s k}^{f g}$ & -0.617 & -0.330 & 9.381 & 0.209 & 0 & 0 & 0.649 & 0.9948 & 1.179 \\
\hline$\delta p_{\text {hip }}^{u s}$ & 0.623 & 0 & 0 & 0 & 0 & 0 & 0 & 0.9985 & $*$ \\
\hline$\theta_{\text {hip }}^{u s}$ & 3.790 & 1.428 & 5.942 & 1.090 & 0.025 & 20.87 & -0.648 & 0.9993 & $*$ \\
\hline$\theta_{s k}^{u s}$ & 6.409 & 0.772 & 2.059 & 3.175 & 0.007 & 9.191 & 0.203 & 0.9956 & $*$ \\
\hline$\theta_{n s k}^{u s}$ & -3.037 & 0.292 & 2.842 & 0.200 & -0.267 & 12.01 & 0.274 & 0.9987 & 0.868 \\
\hline$\delta p_{h i p}^{d s}$ & 0.535 & 0 & 0 & 0 & 0 & 0 & 0 & 0.9981 & $*$ \\
\hline$\theta_{\text {hip }}^{d s}$ & 3.658 & -1.926 & 0.246 & -7.356 & 0.320 & 4.766 & 1.541 & 0.9656 & $*$ \\
\hline$\theta_{s k}^{d s}$ & 0.296 & -10.727 & 0.484 & -5.320 & -0.102 & 17.57 & 10.98 & 0.9830 & * \\
\hline$\theta_{n s k}^{d s}$ & 6.960 & -0.547 & 11.31 & -0.870 & 0.586 & 7.978 & 0.964 & 0.9956 & 2.817 \\
\hline$\delta p_{h i p}^{f \rightarrow u}$ & 0.760 & 0 & 0 & 0 & 0 & 0 & 0 & 0.9993 & $*$ \\
\hline$\theta_{h i p}^{f \rightarrow u}$ & -3.014 & 0.196 & 4.825 & -0.126 & 0.284 & 11.12 & 0.106 & 0.9984 & $*$ \\
\hline$\theta_{s k}^{f \rightarrow u}$ & 0.841 & -0.148 & 6.676 & 1.062 & 0.082 & 6.822 & 0.167 & 0.9091 & $*$ \\
\hline$\theta_{n s k}^{f \rightarrow u}$ & 1.367 & -0.602 & 10.02 & 0.069 & 0.009 & 15.93 & 0.911 & 0.9991 & 3.113 \\
\hline$\delta p_{\text {hip }}^{u \rightarrow f}$ & 0.774 & 0 & 0 & 0 & 0 & 0 & 0 & 0.9981 & $*$ \\
\hline$\theta_{\text {hip }}^{u \rightarrow f}$ & 0.854 & 0.828 & 4.000 & -0.410 & 0.095 & 10.01 & -0.118 & 0.9999 & $*$ \\
\hline$\theta_{s k}^{u \rightarrow f}$ & 2.455 & 0.498 & 2.063 & -1.113 & 0.011 & 24.18 & 0.474 & 0.9822 & $*$ \\
\hline$\theta_{n s k}^{u \rightarrow f}$ & 0.852 & -0.163 & 6.802 & 1.067 & -0.000 & 6.855 & 0.462 & 0.9931 & 2.000 \\
\hline$\delta p_{\text {hip }}^{f \rightarrow d}$ & 0.730 & 0 & 0 & 0 & 0 & 0 & 0 & 0.9990 & $*$ \\
\hline$\theta_{\text {hip }}^{f \rightarrow d}$ & -1.568 & 0.138 & 4.988 & -0.212 & 0.216 & 12.27 & 0.232 & 0.9494 & $*$ \\
\hline$\theta_{s k}^{f \rightarrow d}$ & 0.239 & 6.709 & 0.235 & 15.74 & 0.126 & 13.72 & -6.735 & 0.9382 & $*$ \\
\hline$\theta_{n s k}^{f \rightarrow d}$ & 0.587 & 6.138 & 0.482 & 9.738 & -0.257 & 16.32 & -5.46 & 0.4249 & 10.22 \\
\hline$\delta p_{\text {hip }}^{d \rightarrow f}$ & 0.730 & 0 & 0 & 0 & 0 & 0 & 0 & 0.9996 & $*$ \\
\hline$\theta_{h i p}^{d \rightarrow f}$ & -0.717 & 0.731 & 4.147 & 1.574 & 0.977 & 14.05 & -1.772 & 0.9302 & $*$ \\
\hline$\theta_{s k}^{d \rightarrow f}$ & -0.060 & -9.575 & 0.273 & 4.584 & 0.062 & 17.48 & 9.661 & 0.2506 & $*$ \\
\hline$\theta_{n s k}^{d \rightarrow f}$ & 0.560 & 2.269 & 6.766 & 2.642 & -1.386 & 6.518 & 0.949 & 0.9396 & 11.66 \\
\hline
\end{tabular}


Table 7.4: Optimized parameter values and cost for human functions of running.

\begin{tabular}{|c|c|c|c|c|c|c|c|c|c|}
\hline \multicolumn{10}{|c|}{$y_{2}^{a}=y_{H}(t)$ given in $(2.4)$} \\
\hline * & * & $v_{h i p}$ & $\alpha_{1}$ & $\alpha_{2}$ & $\alpha_{3}$ & $\alpha_{4}$ & $\alpha_{5}$ & Corr & Cost \\
\hline Mean & $\delta p_{\text {hip }}^{\text {stance }}$ & 2.1998 & & - & $*$ & & $*$ & 0.9999 & $*$ \\
\hline * & $\delta p_{\text {hip }}^{\text {flight }}$ & 2.1998 & * & $*$ & * & * & * & 0.9999 & $*$ \\
\hline * & $\theta_{\text {hip }}^{\text {stance }}$ & $*$ & 4.952 & 0.941 & 14.06 & 0.475 & -0.370 & 0.9987 & * \\
\hline * & $\theta_{\text {hip }}^{\text {flight }}$ & * & 13.04 & -0.322 & 2.175 & -2.828 & -0.339 & 0.9960 & * \\
\hline * & $\theta_{s k}^{\text {stance }}$ & * & 11.66 & -0.159 & 3.153 & 6.561 & 0.196 & 0.9304 & $*$ \\
\hline * & $\theta_{s k}^{\text {flight }}$ & $*$ & -6.647 & -0.119 & 24.98 & -0.069 & 0.660 & 0.9904 & * \\
\hline * & $\begin{array}{l}\text { sk } \\
\theta_{n s k}^{\text {stance }}\end{array}$ & * & -1.319 & -0.241 & 23.55 & 0.284 & 1.089 & 0.9630 & * \\
\hline * & $\theta_{n s k}^{f i n h t}$ & * & 0.855 & 0.301 & 25.46 & -0.242 & 0.364 & 0.9910 & 3.02 \\
\hline
\end{tabular}




\section{REFERENCES}

[1] R. Ambrose, H. Aldridge, R. Askew, R. Burridge, W. Bluethmann, M. Diftler, C. Lovchik, D. Magruder, and F. Rehnmark. Robonaut: NASA's space humanoid. IEEE Intelligent Systems and their Applications, 15(4):57 -63, jul/aug 2000.

[2] A. D. Ames. First steps toward automatically generating bipedal robotic walking from human data. In Robotic Motion and Control 2011, volume 422, pages 89116. Springer, 2012.

[3] A. D. Ames, E. A. Cousineau, and M. Powell. Dynamically stable bipedal robotic walking with nao via human-inspired hybrid zero dynamics. In Proceedings of the 15th ACM International Conference on Hybrid Systems: Computation and Control, pages 135-144, New York, NY, USA, 2012. ACM.

[4] G. A. Bekey. Autonomous robots: from biological inspiration to implementation and control. MIT Press, May 2005.

[5] P. Brugger and H. B. Schmiedmayer. Simulating prosthetic gait - lessons to learn. 3(1):64-67, 2003.

[6] R. Burridge, A. Rizzi, and D. Koditschek. Sequential composition of dynamically dexterous robot behaviors. The International Journal of Robotics Research, 18:534-555, 1999.

[7] P. H. Channon, S. H. Hopkins, and D. T. Pham. Derivation of optimal walking motions for a bipedal walking robot. Robotica, 10:165-172, 21992.

[8] C. Chevallereau and Y. Aoustin. Optimal reference trajectories for walking and running of a biped robot. Robotica, 19:557-569, 82001. 
[9] S. H. Collins, A. Ruina, R. Tedrake, and M. Wisse. Efficient bipedal robots based on passive-dynamic walkers. Science, 307:1082-1085, 2005.

[10] S. H. Collins, M. Wisse, and A. Ruina. A three-dimensional passive-dynamic walking robot with two legs and knees. International Journal of Robotics Research, 20:607-615, July 2001.

[11] F. Dierick, M. Penta, D. Renaut, and C. Detrembleur. A force measuring treadmill in clinical gait analysis. 20(3):299-303, Dec. 2004.

[12] C. Endisch and D. Schroder. Funnel-control in robotics: An introduction. Control and Automation, pages 913-9, 2008.

[13] J. W. Grizzle, G. Abba, and F. Plestan. Asymptotically stable walking for biped robots: Analysis via systems with impulse effects. IEEE TAC, 46(1):5164, 2001.

[14] J. W. Grizzle, C. Chevallereau, A. D. Ames, and R. Sinnet. 3D bipedal robotic walking: models, feedback control, and open problems. In IFAC Symposium on Nonlinear Control Systems, pages 505-532, Bologna, 2010.

[15] M. Hirose1 and K. Ogawa. Honda humanoid robots development. Phil. Trans. R. Soc., 365(1850):11-19, Jan 2007.

[16] H. Hirukawa, S. Kajita, K. Harada, S. Hattori, K. Kaneko, M. Morisawa, S. Nakaoka, and F. Kanehiro. A Pattern Generator of Humanoid Robots Walking on a Rough Terrain. IEEE, ICRA, 2007.

[17] Y. Hürmüzlü and D. Marghitu. Rigid body collisions of planar kinematic chains with multiple contact points. Intl. J. of Robotics Research, 13(1):82-92, 1994.

[18] A. Ilchmann, E. Ryan, and S. Trenn. Tracking control: Performance funnels and prescribed transient behaviour. Systems \&f Control Letters, 54:655-670, 2005. 
[19] S. Kolathaya and A. D. Ames. Achieving Bipedal Locomotion on Rough Terrain through Human-Inspired Control. SSRR, pages 3-8, 2012.

[20] P. A. Kramer. The effect on energy expenditure of walking on gradients or carrying burdens. American Journal of Human Biology, 22(4):497-507, 2010.

[21] O. Kwon and J. H. Park. Asymmetric trajectory generation and impedance control forrunning of biped robots. Autonomous Robots, 26(1):47-78, oct 2008.

[22] O. Kyosuke and M. Katsumata. Analytical study of active prosthetic legs. 1(3):548-557, Mar. 2007.

[23] Y.-H. Lee and W.-H. Hong. Effects of shoe inserts and heel height on foot pressure, impact force, and perceived comfort during walking. 36(3):355-362, May 2005.

[24] F. Matsuno and S. Tadokoro. Rescue robots and systems in japan. pages 12 20 , Oct 2004 .

[25] T. McGeer. Passive dynamic walking. Intl. J. of Robotics Research, 9(2):62-82, Apr. 1990.

[26] R. M. Murray, Z. Li, and S. S. Sastry. A Mathematical Introduction to Robotic Manipulation. CRC Press, Boca Raton, 1994.

[27] J. K. Rai, R. P. Tewari, and D. Chandra. Hybrid control strategy for robotic leg prosthesis using artificial gait synthesis. 1(1):44-50, 2009.

[28] J. Rose and J. G. Gamble. Human Walking. Lippincott Williams \& Wilkins, Philadelphia, Dec. 2005.

[29] S. S. Sastry. Nonlinear Systems: Analysis, Stability and Control. Springer, New York, 1999. 
[30] R. Sinnet, M. Powell, S. Jiang, and A. D. Ames. Compass gait revisited: A human data perspective with extensions to three dimensions, 2011. Submitted for publication, available upon request.

[31] R. Tedrake, I. R. Manchester, M. Tobenkin, and J. W. Roberts. Lqr-trees: Feedback motion planning via sums of squares verification. International Journal of Robotics Research, pages 1038-1052, 2010.

[32] D. G. Thelen and F. C. Anderson. Using computed muscle control to generate forward dynamic simulations of human walking from experimental data. 39(6):1107 -1115, Jan. 2006.

[33] E. R. Westervelt, J. W. Grizzle, C. Chevallereau, J. H. Choi, and B. Morris. Feedback Control of Dynamic Bipedal Robot Locomotion. CRC Press, 2007.

[34] E. R. Westervelt, J. W. Grizzle, and D. E. Koditschek. Hybrid zero dynamics of planar biped walkers. IEEE TAC, 48(1):42-56, 2003.

[35] D. Winter. Biomechanics and Motor Control of Human Movement. WileyInterscience, New York, 2 edition, 1990.

[36] Y. Xiang, J. S. Arora, S. Rahmatalla, and K. Abdel-Malek. Optimization-based dynamic human walking prediction:one step formulation. 79(667 - 695):291-305, Feb 2009.

[37] Y. Xiang, H.-J. Chung, J. H. Kim, R. Bhatt, and S. Rahmatalla. Predictive dynamics an optimization-based novel approach for human motion simulation. Biomechanical Application, 41(4):465-479, 2010.

[38] H. Zhao, M. Powell, and A. D. Ames. Human-inspired motion primitives and transitions for bipedal robotic locomotion in diverse terrain. Optimal Control Applications and Methods, 35:730-755, 2013. 
[39] H. Zhao, N. S. Yadukumar, and A. D. Ames. Bipedal robotic running with partial hybrid zero dynamics and human-inspired optimization. In IROS, 2012 IEEE/RSJ International Conference on, pages 1821-1827. IEEE, 2012. 